Sabine Boerner, Hannah Keding \& Hendrik Hüttermann*

\title{
Gender Diversity und Organisationserfolg - Eine kritische Bestandsaufnahme
}

\section{Gender Diversity and Performance - \\ A Critical Review}

\section{Zusammenfassung}

Diversity Management hat sich in der Personalpolitik deutscher Unternehmen als unverzichtbares Element etabliert. Die Motive hierfür sind unterschiedlich und reichen von politisch-normativer Notwendigkeit bis zur Erwartung ökonomischer Vorteile. Vor diesem Hintergrund analysiert der Beitrag die Ergebnisse der aktuellen internationalen Forschung zum Zusammenhang zwischen Gender Diversity und Organisationserfolg. Dabei werden u.a. erfolgskritische Randbedingungen dieses Zusammenhangs betrachtet, darunter auch Ansätze des Managements von Diversity. Es zeigt sich, dass die einschlägige Forschung derzeit keine Schlüsse auf eine generelle ökonomische Vorteilhaftigkeit von Gender Diversity zulässt; auch konnten bisher nur wenige Randbedingungen als erfolgskritisch identifiziert werden. Defizite der bisherigen Forschung bestehen vor allem in der unzureichenden theoretischen Fundierung und methodischen Heterogenität der empirischen Untersuchungen; überdies sind Studien im Kontext deutschsprachiger Unternehmen ausgesprochen selten. Der Beitrag diskutiert Implikationen für Forschung und Praxis des Managements von Gender Diversity.

Keywords: Gender Diversity, Erfolg, Forschungsergebnisse, Bestandsaufnahme

\footnotetext{
* Prof. Dr. Sabine Boerner, Jg. 1963, Professorin an der Universität Konstanz, Lehrstuhl für Management, insbesondere Strategie und Führung, Postanschrift: Universitätsstraße 10, 78464 Konstanz, E-Mail: sabine.boerner@uni-konstanz.de. Hannah Keding, Jg. 1987, Studentin im Master-Studiengang Politik- und Verwaltungswissenschaft an der Universität Konstanz, Postanschrift: Universitätsstraße 10, 78464 Konstanz, E-Mail: hannah.keding@uni-konstanz,de. Hendrik Hüttermann, M.A., Jg. 1984, Wissenschaftlicher Mitarbeiter an der Universität Konstanz, Lehrstuhl für Management, insbesondere Strategie und Führung, Postanschrift: Universitätsstraße 10, 78464 Konstanz, E-Mail: hendrik.huettermann@uni-konstanz,de.
} 


\section{Summary}

In the context of human resource management in German organizations, diversity management has been established as an indispensible element. The reasons for diversity management are various, ranging from a response to political or normative needs to the expectation of positive economic effects. Against this background, the paper presents empirical results of the current international research on the relationship between gender diversity and organizational performance, including moderators of this relationship. Our analysis reveals that positive economic main effects of gender diversity cannot be confirmed; moreover, only few moderators of the diversity-performance relationship could be identified. Extant research suffers from a lack of consistent theoretical reasoning and methodological heterogeneity; moreover, studies in the German context are particularly rare. The paper provides implications for further research on gender diversity and practical implications for gender diversity management.

Keywords: gender diversity, performance, state-of-science, review JEL-classification: M12, M50, M54

Dieser Artikel wurde zur Publikation angenommen und hat den vollständigen ReviewProzess durchlaufen, befindet sich jedoch noch nicht in der von der Zeitschrift erstellten finalen Formatierung. Dies kann zu kleineren Abweichungen zwischen dieser und der Druck-Version (https://doi.org/10.1007/BF03373689) führen.

Der Artikel kann wie folgt zitiert werden: Boerner, S., Keding, H., \& Huettermann, H. (2012). Gender Diversity und Organisationserfolg: Eine kritische Bestandsaufnahme. Schmalenbachs Zeitschrift für betriebswirtschaftliche Forschung, 64, 37-70. 


\section{Gender Diversity und Organisationserfolg - \\ Eine kritische Bestandsaufnahme}

\section{Einleitung}

Organisationen des privaten und des öffentlichen Sektors verzeichnen seit mehreren Jahren eine wachsende Heterogenität ihrer Belegschaften im Hinblick auf unterschiedliche Merkmale wie Geschlecht, Alter, ethnische Herkunft oder Bildung ${ }^{1}$. Dieses in der organisationswissenschaftlichen Forschung unter dem Begriff „Diversity“ untersuchte Phänomen hat sowohl exogene als auch endogene Ursachen: Einerseits bewirken Entwicklungen wie demographischer Wandel oder Globalisierung eine zunehmende gesellschaftliche Heterogenisierung, die sich in organisationalen Belegschaften widerspiegelt. Andererseits wird Diversity in Organisationen unter dem Stichwort „Diversity Management" gezielt gefördert, etwa durch eine entsprechende Personalauswahl und entwicklung ${ }^{2}$. Diversity Management umfasst entsprechend zum einen Anstrengungen zur Unterstützung der Zusammenarbeit heterogener Belegschaften, zum anderen werden darunter Maßnahmen zur gezielten Förderung der Heterogenität von Belegschaften verstanden ${ }^{3}$. Auch in deutschen Unternehmen ist Diversity Management mittlerweile weit verbreitet ${ }^{4}$. So verfügen beispielsweise die Automobilhersteller Daimler und Volkswagen, der Energiekonzern Eon und der Softwarehersteller IBM über eigene „Diversity Management“-Konzepte. Daimler-Chef Dieter Zetsche bezeichnet ein effektives Diversity Management als „sozial und geschäftlich sinnvoll, ja notwendig“5. Dies gilt nicht nur für den privaten, sondern auch für den öffentlichen Sektor, wo beispielsweise „Frauen im Rahmen der rechtlichen Möglichkeiten vorrangig berücksichtigt“" werden ${ }^{6}$. Dabei steht speziell Gender Diversity im Zentrum der genannten Diversity Management-Konzepte - so wird Gender in der Personalpolitik deutscher wie internationaler Unternehmen als die wichtigste Diversity-Dimension betrachtet ${ }^{7}$.

\footnotetext{
${ }^{1}$ Vgl. Jackson/Joshi/Erhardt (2003).

${ }^{2}$ Vgl. Williams/O'Reilly (1998); van Knippenberg/Schippers (2007).

${ }^{3}$ Vgl. z.B. Klaffke (2009); Süß (2009); Köppel (2010).

${ }^{4}$ Vgl. Köppel (2010), S. 23.

${ }^{5}$ Deckstein (2008), S. 23.

${ }^{6}$ HMTH (2010).

${ }^{7}$ Vgl. Süß (2008), S. 417; Krell (2009), S. 146; Köppel (2010), S. 25.
} 
Die Motive für das Management von Gender Diversity sind unterschiedlich und reichen von politisch-normativen Notwendigkeiten bis zu der Erwartung ökonomischer Vortei$1 e^{8}$. Die gezielte Förderung geschlechter-heterogener Belegschaften kann zum einen als Widerspiegelung der aktuellen politisch-normativen gesellschaftlichen Diskussion interpretiert werden. Vor allem der Gedanke der Gleichberechtigung bzw. Chancengleichheit der Geschlechter (,moral justice case“) konkretisiert sich in (teilweise gesetzlich verankerten) Normen zur Förderung von Frauen in vielen Organisationsbereichen (z.B. Frauenquoten in Aufsichtsräten und Vorständen ${ }^{9}$; Verbot geschlechtsbezogener Diskriminierung bei Personalentscheidungen ${ }^{10}$ ). Dieselbe Diskussion führt dazu, bisher „typische“ Frauenberufe (z.B. im Pflege- und Erziehungsbereich) auch für Männer attraktiv zu machen und somit durch die Förderung der „Männerquote“ die Geschlechterheterogenität zu erhöhen. In Erfüllung gesellschaftlicher Erwartungen und rechtlicher Vorgaben sind Organisationen daher interessiert, Gender-Diversity zu etablieren und zu unterstützen.

Zum anderen herrscht in vielen Organisationen die Überzeugung vor, dass Gender Diversity einen ökonomischen Erfolgsfaktor darstellt („business case“). So werden in der einschlägigen deutschsprachigen Literatur positive Erwartungen an (Gender) Diversity nicht nur unter der Perspektive von Anti-Diskriminierung und Fairness diskutiert, sondern auch unter den Stichworten „Lern- und Effektivitätsparadigma““11. Entsprechend bestätigen Untersuchungen in deutschen Unternehmen, dass Manager sich von Diversity-Management auch konkrete ökonomische Vorteile versprechen, etwa bei der Internationalisierung, bei der Schaffung und Nutzung von Mitarbeiterpotentialen, beim Marketing und bei der internationalen Zusammenarbeit ${ }^{12}$. Ein weiteres entscheidendes Argument ist zudem der mit dem demographischen Wandel einhergehende Fachkräftemangel auf dem deutschen Arbeitsmarkt, aufgrund dessen Unternehmen in zunehmendem Maße darauf angewiesen sind, die Potentiale der Beschäftigten beider Geschlechter zu nutzen ${ }^{13}$.

Eine Verbindung zwischen der politisch-normativen und der ökonomischen Argumentation kommt letztlich in der Erwartung zum Ausdruck, über die Förderung von Gender

\footnotetext{
${ }^{8}$ Vgl. Krell/Ortlieb/Sieben (2011).

${ }^{9}$ Vgl. Deutscher Bundestag (2011); Telekom (2010).

${ }^{10} \mathrm{Vgl}$. etwa Allgemeines Gleichbehandlungsgesetz (2006).

${ }^{11}$ Vgl. Voigt/Wagner (2007), S. 22; Klaffke (2009), S. 143.

12 Süß (2008), S. 421; Köppel (2010), S. 26.

${ }^{13}$ Vgl. Bundesagentur für Arbeit (2011).
} 
Diversity Reputationsgewinne am Markt zu erzielen - eine Motivation, die unter dem Stichwort „Marktzugangsparadigma“ diskutiert wird ${ }^{14}$. So werben Unternehmen gezielt mit (Gender)-Diversity, Frauenförderung oder Familienfreundlichkeit um potenzielle Mitarbeiter und Kunden ${ }^{15}$.

Angesichts der Aktualität der Thematik ist zu erwarten, dass der Trend zur gezielten Förderung geschlechtsbezogener Heterogenität zukünftig eher zu- als abnehmen wird. Vor diesem Hintergrund analysiert der vorliegende Beitrag die Ergebnisse einschlägiger empirischer Untersuchungen der internationalen Diversity-Forschung. Im Zentrum steht dabei die Frage, welche (positiven oder negativen) Effekte Gender Diversity auf den Organisationserfolg ausübt. Die kritische Analyse performanzrelevanter Effekte von Gender Diversity erscheint einerseits angesichts der Anpassung der Unternehmen an politisch-normative Notwendigkeiten sinnvoll, da diese bedeutende Implikationen für die ökonomische Leistungsfähigkeit von Organisationen mit sich bringen kann. Andererseits dient sie der Überprüfung der zitierten ökonomischen Erwartungen an eine aktive Förderung von Gender Diversity.

Die Frage, ob und unter welchen Bedingungen sich Gender Diversity tatsächlich positiv auf den Organisationserfolg auswirkt, wurde in zahlreichen Arbeiten untersucht, insbesondere häufiger als zum Beispiel die Effekte von Altersdiversität oder ethnischer Diversität ${ }^{16}$. Dabei wird Gender Diversity verstanden als Grad, in dem Organisationen oder organisationale Teams in Bezug auf das Geschlecht ihrer Mitglieder heterogen zusammengesetzt sind. Da Diversity zumeist auf der Ebene von Teams (z.B. Arbeitsgruppen, Abteilungen, Filialen) analysiert wird, beziehen sich die Aussagen zum Organisationserfolg in der Regel ebenfalls auf die Teamebene - eine Ausnahme stellen Studien über Top Management Teams und Aufsichtsräte dar, bei denen der Erfolg auf der Organisationsebene erhoben wird. Entsprechend werden die Variablen „Organisationserfolg“ oder „organisationale Performanz“ im Folgenden in einem weiteren Sinne verstanden, der auch die Teamebene einschließt.

Literatur-Reviews und Meta-Analysen zeichnen ein heterogenes Bild der bestehenden Empirie. So kommen Bowers/Pharmer/Salas (2000) in ihrer Meta-Analyse von 13 Studien zur Wirkung von Diversity auf Teamebene zu dem Ergebnis, dass die ermittelten

\footnotetext{
${ }^{14}$ Vgl. Voigt/Wagner (2007), S. 22; Klaffke (2009), S. 142.

${ }^{15}$ Vgl. etwa Allianz (2011); SAP (2011); Deutsche Bahn (2010).

${ }^{16} \mathrm{Vgl}$. Jackson/Joshi/Erhardt (2003), S. 804.
} 
Effektgrößen zu klein sind, um tragfähige Schlüsse zur Wirkung von Gender Diversity auf Performanz zuzulassen ${ }^{17}$. Ebenfalls in einer Meta-Analyse werten Webber/Donahue (2001) 24 Studien zu Diversity auf Teamerfolg aus und können ebenso wenig einen signifikanten direkten Effekt von Gender Diversity feststellen ${ }^{18}$. Zu dem gleichen Ergebnis kommen Joshi/Roh (2009) in ihrer Meta-Analyse von 39 Studien. Während die existierenden Meta-Analysen somit keinen signifikant positiven Zusammenhang zwischen Gender Diversity und Organisationserfolg bestätigen können, sind die Resultate der bestehenden Literatur-Reviews - nicht zuletzt aufgrund ihrer differenzierteren und weniger aggregierenden Analyse - weniger einheitlich ${ }^{19}$. So berichten Jackson/Joshi/Erhardt (2003), Shore et al. (2009) und Terjesen/Sealy/Singh (2009) jeweils inkonsistente Befunde ${ }^{20}$, während Kochan et al. (2003) keinen signifikanten Effekt feststellen ${ }^{21}$; im Gegensatz dazu weist der jüngste Literatur-Review von Pitts/Wise (2010) einen positiven Zusammenhang zwischen Gender Diversity und Organisationserfolg aus ${ }^{22}$. Eine aktuelle zusammenfassende Auswertung der Ergebnisse dieser Übersichtsstudien fehlt bislang in der Forschungsliteratur; darüber hinaus sind verschiedene Primärstudien bislang nicht in Meta-Analysen oder Literatur-Reviews berücksichtigt worden. Vor diesem Hintergrund versteht sich unsere Analyse auch im Sinne eines Reviews als Beitrag zur Erschließung des aktuellen Standes der internationalen Forschung zum Zusammenhang zwischen Gender Diversity und Organisationserfolg.

Der Beitrag ist wie folgt gegliedert: Im ersten Schritt werden die grundlegenden Theoriestränge dargestellt, die mögliche Zusammenhänge zwischen Gender Diversity und Organisationserfolg begründen. Im zweiten Schritt werden aktuelle empirische Ergebnisse zum direkten Einfluss von Gender Diversity auf den Organisationserfolg dargestellt. Dabei lassen sich zwar vereinzelt positive, überwiegend jedoch inkonsistente und nicht-signifikante Zusammenhänge nachweisen. Zusätzlich werden daher Befunde über Moderator-Variablen berichtet, die den jeweiligen Zusammenhang zwischen Gender Diversity und Organisationserfolg verstärken oder reduzieren; auch hier zeigen sich teilweise widersprüchliche Ergebnisse. Zusammenfassend erlauben die Ergebnisse der aktuellen empirischen Forschung damit keine Schlüsse auf eine unmittelbare ökonomische Vorteilhaftigkeit von Gender Diversity. Zugleich wird erkennbar, dass bislang nur

\footnotetext{
${ }^{17} \mathrm{Vgl}$. Bowers/Pharmer/Salas (2000).

${ }^{18}$ Vgl. Webber/Donahue (2001).

${ }^{19} \mathrm{Vgl}$. Joshi/Roh (2009).

${ }^{20}$ Vgl. Jackson/Joshi/Erhardt (2003); Shore et al. (2009); Terjesen/Sealy/Singh (2009).

${ }^{21}$ Vgl. Kochan et al. (2003).

${ }^{22}$ Vgl. Pitts/Wise (2010).
} 
wenige erfolgsrelevante Kontextfaktoren bestätigt werden konnten - selbst die Wirkungen von Diversity-Management sind nicht eindeutig positiv. Im dritten Schritt werden die ausgewählten Studien hinsichtlich ihrer methodischen Vorgehensweise kritisch betrachtet. Dabei wird sich zeigen, dass die Studien eine starke Heterogenität aufweisen, die vermutlich eine Teilerklärung für ihre Widersprüchlichkeit darstellt. Der Beitrag schließt mit Implikationen für Forschung und Praxis im Kontext von Gender Diversity und Gender Diversity Management.

\section{Theoretische Argumentation: Erwartungen zur Wirkung von Gender Diversity auf den Organisationserfolg}

Van Knippenberg/Schippers (2007, S. 516) definieren Diversity als ,a characteristic of social grouping that reflects the degree to which objective or subjective differences exist between group members"23. Diese subjektiven oder objektiven Unterschiede werden in der Regel klassifiziert nach beziehungsorientierten („,relations-oriented”; z.B. Alter, Sprache, Geschlecht) vs. aufgabenorientierten (,task-related“; z.B. Bildungsgrad, betriebliche Funktion) Merkmalen, die entweder direkt beobachtbar (,surface-level“) oder nicht direkt beobachtbar sind (,,deep-level“). Gender Diversity wird dabei als eine Form von Diversity eingestuft, die auf Anhieb sichtbar und beziehungsorientiert (anstatt aufgabenorientiert) ist und mit Unterschieden in den Werten und Einstellungen der Teammitglieder einhergeht ${ }^{24}$. In der Diversity-Forschung wird die Kategorie Gender in der Regel nicht gesondert untersucht, sondern zusammen mit ethnischer Herkunft und Alter als typischer Vertreter von sichtbaren, beziehungsorientierten Diversity-Merkmalen.

Im Hinblick auf die Effekte von (Gender) Diversity im Organisationskontext lassen sich in der sozialpsychologischen Forschung zwei widersprüchliche theoretische Perspektiven unterscheiden ${ }^{25}$. Die „Information/Decision-Making“- Perspektive postuliert, dass heterogene Teams über ein größeres Potential an Erfahrungen, Informationen und Perspektiven verfügen als homogene Teams. Dieser erweiterte Ressourcenpool führt letztlich zu besseren Ideen und Entscheidungen und damit überlegenen Arbeitsergebnis-

\footnotetext{
${ }^{23}$ van Knippenberg/Schippers (2007), S. 516.

${ }^{24}$ Vgl. z.B. Webber/Donahue (2001), S. 144; Jackson/Joshi/Erhardt (2003), S. 802; Leonard/Levine/Joshi (2004), S. 732.; Gonzalez/Denisi (2009), S. 22; Joshi/Roh (2009), S. 600.

${ }^{25} \mathrm{Vgl}$. Williams/O'Reilly (1998); van Knippenberg/Schippers (2007), S. 517.
} 
$\operatorname{sen}^{26}$. So argumentieren Hirschfeld et al. (2005), Gender Diversity erweitere die mentalen Ressourcen von Teams, weil Frauen andere mentale Stärken besitzen als Männer. Konkret seien Frauen Männern überlegen in Bezug auf verbale Fähigkeiten, feinmotorische Fähigkeiten, Wahrnehmungsgeschwindigkeit und die Dekodierung nonverbaler Kommunikation $^{27}$. Männer besäßen demgegenüber Vorteile in Bezug auf visuelles und räumliches Vorstellungsvermögen und verschiedene mathematische Fähigkeiten. Ein gemischt-geschlechtliches Team habe daher eine größere Bandbreite an mentalen Ressourcen zur Verfügung. Analog zitieren Wegge et al. (2008) Verhaltensunterschiede zwischen Männern und Frauen, deren Kombination die Überlegenheit geschlechtergemischter Teams bezgl. Performanz erklärt ${ }^{28}$ : Männliches Verhalten wird als durchsetzungsfähig, eigensinnig und aufgabenbezogen beschrieben; Frauen verhielten sich demgegenüber freundlich, zustimmend und prozessorientiert. Teams, in denen Männer und Frauen arbeiten, würden entsprechend über ein größeres Verhaltensrepertoire verfügen als geschlechtshomogene Teams.

Im Gegensatz dazu sind nach der „Social Categorization“-Perspektive negative Auswirkungen von Diversity zu erwarten ${ }^{29}$ : Wahrgenommene Unterschiede zwischen Teammitgliedern führen zu Prozessen der sozialen Kategorisierung, die die Bildung von sogenannten In- und Outgroups innerhalb heterogener Teams begünstigen. Dabei werden ähnliche Teammitglieder der eigenen Ingroup zugeordnet, die als vertrauenswürdiger gilt und deren Mitglieder positiver beurteilt werden als Mitglieder der Outgroup ${ }^{30}$. In Übereinstimmung mit der Similarity-Attraction-Theorie ergibt sich daraus die Konsequenz, dass Teammitglieder eher zur Kooperation mit Mitgliedern der eigenen in-group bereit sind, während sich mit Mitgliedern der out-group Kommunikations- und Kooperationsprobleme ergeben ${ }^{31}$. Diese Kooperationsdefizite können sich wiederum negativ auf die Performanz auswirken ${ }^{32}$. So verweisen etwa Joshi/Roh (2009) darauf, dass speziell in solchen Kontexten, in denen Frauen unterrepräsentiert sind, die soziale Kategorie „Gender“ eher salient wird und sich mit der Zuschreibung von geringerer Kompetenz für Frauen verbindet ${ }^{33}$.

\footnotetext{
${ }^{26}$ Vgl. van Knippenberg/Schippers (2007).

${ }^{27} \mathrm{Vgl}$. Hirschfeld et al. (2005), S. 899.

${ }^{28}$ Vgl. Wegge et al. (2008), S. 1303.

${ }^{29}$ Vgl. van Knippenberg/De Dreu/Homan (2004).

${ }^{30}$ Vgl. Brewer (1979); Tajfel/Turner (1986); Turner et al. (1987).

${ }^{31}$ Vgl. Byrne (1971); Berscheid/Walster (1978).

${ }^{32}$ Vgl. Richard et al. (2004); Gonzalez/Denisi (2009); Joshi/Roh (2009).

${ }^{33} \mathrm{Vgl}$. Joshi/Roh (2009), S. 607.
} 
Ähnlich widersprüchlich sind die Erwartungen zum Einfluss von Diversity auf Performanz in der organisationstheoretischen Literatur ${ }^{34}$. Eine positive Wirkung Gendergemischter Aufsichtsräte wird etwa im Sinne der Signaling Theory ${ }^{35}$ und der Theorie des Neo-Institutionalismus ${ }^{36}$ erwartet: Gender-diverse Aufsichtsräte signalisieren der Unternehmensumwelt erstens die Erfüllung sozialer Normen und zweitens die Fähigkeit, sich auf gegebenenfalls „diverse“ Stakeholder und Märkte einstellen zu können, was über die Erhöhung der Reputation zur Steigerung der Unternehmensperformanz führen kann. Im Kontext der Resource Dependence-Theorie ${ }^{37}$ und der Behavioral Theory of the Firm ${ }^{38}$ wird argumentiert, dass heterogene Teams über ein größeres Humankapital verfügen als homogene Teams ${ }^{39}$. Dies kann zur Optimierung interner Entscheidungsprozesse führen. Im Hinblick auf mögliche negative Effekte von Gender Diversity auf Performanz postuliert die Humankapital-Theorie jedoch, dass Gender-gemischte Aufsichtsräte zwar einzigartiges Humankapital schaffen, die Effekte dieses Humankapitals auf die Performanz jedoch sowohl positiv als auch negativ sein können ${ }^{40}$.

Zusammenfassend sind aus theoretischer Sicht sowohl positive als auch negative Effekte von Gender Diversity auf den Organisationserfolg zu erwarten. Shore et al. (2009) verweisen in ihrem Review allerdings darauf, dass die meisten empirischen Studien zu den Effekten von Gender Diversity theoretische Perspektiven formulieren, die negative Effekte - etwa im Sinne der „Social Categorization“-Perspektive - vorhersagen ${ }^{41}$. Vor diesem Hintergrund werden im Folgenden die empirischen Befunde zu den Effekten von Gender Diversity beleuchtet.

\section{Empirische Befunde zur Wirkung von Gender Diversity auf den Organisations- erfolg}

Ausgehend von dem bisher umfangreichsten einschlägigen Literatur-Review $\mathrm{zu}$ den Effekten von Gender Diversity auf den Organisationserfolg von Kochan et al. (2003) wurde das Jahr 2003 als Referenz für die systematische Analyse der internationalen

\footnotetext{
${ }^{34}$ Vgl. Carter et al. (2010).

35 Vgl. Certo (2003).

${ }^{36}$ Vgl. Powell/DiMaggio (1991).

${ }^{37}$ Vgl. Salancik/Pfeffer (1978).

${ }^{38}$ Vgl. Cyert/March (1963).

${ }^{39}$ Vgl. Miller/del Carmen Triana (2009), S. 758.

${ }^{40}$ Vgl. Terjesen/Sealy/Singh (2009), S. 330.

${ }^{41}$ Vgl. Shore et al. (2009).
} 
Literatur gewählt ${ }^{42}$. Die Recherche wurde mithilfe der Datenbanken Business Source Premier, PsycINFO, WISO-NET und Social Sciences Citation Index durchgeführt. Kriterium für die Berücksichtigung von Studien war, dass der Zusammenhang zwischen der unabhängigen Variablen Gender Diversity und der abhängigen Variablen Organisationserfolg im weiteren Sinne untersucht wurde. Weitere Arbeiten wurden per „Schneeballverfahren“" ausfindig gemacht, d.h. die in der Datenbankrecherche identifizierten Publikationen wurden systematisch nach Referenzen zu themenbezogenen empirischen Studien durchsucht, um zu gewährleisten, dass keine Studie übersehen wurde. Insgesamt erbrachte die Suche 18 Studien (darunter fünf Literatur-Reviews und eine Metanalyse), die der nachfolgenden Analyse zugrunde liegen (vgl. Tabelle 1).

\#Tabelle 1 bitte hier einfügen\#

Im Folgenden werden die Befunde der identifizierten Studien analysiert. Dabei werden zunächst direkte Zusammenhänge zwischen Gender Diversity und Organisationserfolg berichtet, um zu überprüfen, ob sich ein genereller Trend bezüglich positiver oder negativer Effekte erkennen lässt. Im Anschluss wird die Frage geklärt, ob die Effektivität von Gender-Diversity möglicherweise kontextspezifisch variiert. Hierzu werden solche Studien betrachtet, die explizit moderierende Effekte der Diversity-PerformanceBeziehung untersuchen.

Die Analyse wird zum einen zeigen, dass die theoretische Argumentation innerhalb der Studien mitunter wenig schlüssig bzw. widersprüchlich ausfällt. Zum anderen weisen die verschiedenen Arbeiten widersprüchliche Befunde auf, was vermutlich auch mit ihrer starken methodischen Heterogenität zusammenhängt. So werden beispielsweise unterschiedliche Operationalisierungen für Gender Diversity als unabhängige Variable und für Performanz als abhängige Variable verwendet. Während theoretische Defizite jeweils im Zuge der Darstellung der betreffenden Studien erörtert werden, erfolgt eine Diskussion methodischer Unterschiede in einem abschließenden Kapitel.

\subsection{Direkte Effekte}

${ }^{42}$ Vgl. Kochan et al. (2003). 
Nur drei der hier referierten 18 Arbeiten weisen eindeutige direkte positive Effekte von Gender Diversity auf den Organisationserfolg aus. So berücksichtigen Pitts/Wise (2010) in ihrem Literatur-Review zu den Effekten von Diversity im öffentlichen Sektor 89 Studien, von denen sich 44 mit Gender Diversity beschäftigen ${ }^{43}$. Nur drei dieser Arbeiten untersuchen den Zusammenhang zwischen Gender Diversity und Performanz empirisch; diese Studien bestätigen die erwarteten positive Effekte von Gender Diversity auf verschiedene Performanz-Maße. Eine Untersuchung in 67 US-Bundesbehörden zeigt, dass Angestellte geschlechterdiverser Organisationen die Organisationsleistung positiver beurteilen als Angestellte von Organisationen, in denen Frauen oder Männer jeweils eine deutliche Mehrheit darstellen ${ }^{44}$. Herring (2009) bestätigt in seiner Untersuchung von organisationaler Diversity in 506 For-Profit-Unternehmen positive Zusammenhänge zwischen Gender Diversity und verschiedenen Performanz-Indikatoren ${ }^{45}$.

Dagegen bestätigt Choi (2009) in seiner Studie von 96 koreanischen Arbeitsgruppen seine Erwartung, dass Gender Diversity negative Effekte auf das Organizational Citizenship Behavior der Gruppenmitglieder hat ${ }^{46}$.

Die Mehrzahl der berücksichtigten Literatur-Reviews verweist jedoch auf inkonsistente Befunde. So fassen Shore et al. (2009) als Ergebnis ihres Literatur-Reviews der Jahre 2000 bis 2007 zusammen, dass die meisten Studien widersprüchliche Ergebnisse zum Einfluss von Gender Diversity auf Performanz aufweisen ${ }^{47}$. In ihrem Review von 400 Literaturquellen zu Studien über Gender Diversity in Aufsichtsräten berichten Terjesen/Sealy/Singh (2009) ebenfalls inkonsistente Ergebnisse ${ }^{48}$. Auch Jackson/Joshi/Erhardt (2003) beschreiben in ihrem Literatur-Review, der 63 zwischen 1997 und 2002 erschienene Feldstudien zur Wirkung von Gender Diversity auf Organisationserfolg umfasst, teilweise positive, teilweise negative und teilweise insignifikante Ergebnisse $^{49}$.

Ein großer Teil der Studien berichtet nicht-signifikante direkte Effekte. Wie frühere Meta-Analysen ${ }^{50}$ und der Review von Kochan et al. (2003) ${ }^{51}$ bestätigt auch die aktuellste

\footnotetext{
${ }^{43}$ Vgl. Pitts/Wise (2010).

${ }^{44}$ Vgl. Choi/Rainey (2010).

${ }^{45}$ Vgl. Herring (2009).

46 Vgl. Choi (2009).

${ }^{47}$ Vgl. Shore et al. (2009).

${ }^{48}$ Vgl. Terjesen/Sealy/Singh (2009).

${ }^{49} \mathrm{Vgl}$. Jackson/Joshi/Erhardt (2003).

${ }^{50}$ Vgl. Bowers/Pharmer/Salas (2000); Webber/Donahaue (2001).
} 
Meta-Analyse von Joshi/Roh (2009) dieses Ergebnis ${ }^{52}$ : Bei 39 einbezogenen empirischen Studien zeigt sich kein signifikanter Haupteffekt von Gender Diversity auf die Organisationsleistung. Auch die Primärstudien, die der vorliegenden Analyse zu Grunde liegen, weisen zum Teil insignifikante oder keine Haupteffekte von Gender Diversity auf die Organisationsleistung auf. Dies gilt u.a. für die Arbeit von Carter et al. (2010), die ihre Erwartung positiver Effekte von Gender Diversity auf die Unternehmensperformanz in einer Studie an 641 US-amerikanischen Aufsichtsräten nicht bestätigen können $^{53}$. Da der Fokus der übrigen Primärstudien auf Moderationseffekten liegt, werden diese im folgenden Abschnitt 3.2 dargestellt ${ }^{54}$.

Zusätzlich zu den oben berichteten linearen Zusammenhängen wurden vereinzelt nichtlineare Zusammenhänge zwischen Gender Diversity und Organisationserfolg untersucht. Shore et al. (2009) berichten in ihrem Review unter anderem eine invers-Uförmige Beziehung zwischen Gender Diversity auf Organisationsebene und Firmenleistung $^{55}$; dagegen kann eine invers-U-förmige Beziehung zwischen Gender Diversity und Leistung in einer Studie an 153 US-amerikanischen Banken nicht eindeutig nachgewiesen werden ${ }^{56}$.

Die dargestellten inkonsistenten empirischen Befunde weisen darauf hin, dass Stärke und Richtung des Einflusses von Gender Diversity auf Organisationserfolg möglicherweise von den jeweiligen Randbedingungen abhängen. Aus diesem Grund werden im Folgenden die Ergebnisse von Studien berichtet, die entsprechende Moderationseffekte untersuchen.

\subsection{Moderationseffekte}

Ein großer Teil der identifizierten Studien untersucht Moderatoren des Zusammenhangs zwischen Gender Diversity und Performanz. Ein Teil dieser Moderatoren wurde allerdings jeweils nur in einer einzelnen Primärstudie thematisiert. Beispielsweise untersuchen Gonzalez/Denisi (2009) das Diversity Climate als Moderator, während

\footnotetext{
${ }^{51}$ Vgl. Kochan et al. (2003).

${ }^{52} \mathrm{Vgl}$. Joshi/Roh (2009).

${ }^{53}$ Vgl. Carter et al. (2010).

${ }^{54} \mathrm{Vgl}$. Gonzalez/Denisi (2009); Kochan et al. (2003); Leonard/Levine/Joshi (2004); Shapcott/Carron/Burke/Bradshaw/Estabrooks (2006); Shaw (2004).

${ }^{55} \mathrm{Vgl}$. Shore et al. (2009).

${ }^{56}$ Vgl. Richard/Barnett/Dwyer/Chadwick (2004).
} 
Richard/Ford/Ismail (2006) den moderierenden Einfluss der organisationalen Kontrollspanne beleuchten. Da hinsichtlich dieser Moderatoren somit kein Vergleich möglich ist, werden die entsprechenden Moderatoren der Primärstudien lediglich im Anhang erfasst.

Im Folgenden werden nur solche Moderatoren beschrieben, die in Meta-Analysen oder Literatur-Reviews als wirksam bestätigt werden konnten. Dabei werden Ergebnisse etwaiger Einzel- bzw. Primärstudien, die dieselben Moderatoren untersuchen, jedoch nicht in der jeweiligen Meta-Analyse bzw. dem jeweiligen Review berücksichtigt wurden, ergänzend präsentiert (unabhängig davon, ob sie die Befunde der Meta-Analysen bzw. Literatur-Reviews bestätigen oder nicht). Die identifizierten Moderatoren lassen sich in drei Kategorien einordnen: (1) Organisationale Einflussfaktoren (Beschäftigungssektor, Geschlechterverhältnis in der jeweiligen Branche, Organisationskultur), (2) Teameigenschaften (Team-Interdependenz, Dauer der Zusammenarbeit im Team) und (3) Diversity-Management (HR-Praktiken, Geschlecht der Führungskraft).

\subsubsection{Organisationale Einflussfaktoren}

Joshi/Roh (2009) konnten in ihrer Meta-Analyse zeigen, dass der Beschäftigungssektor für die Wirkung der untersuchten beziehungsorientierten Diversity-Merkmale (u.a. Gender) eine Rolle spielt: Bei zunehmender Gender Diversity zeigt sich eine verbesserte Leistung im Dienstleistungssektor, jedoch eine verschlechterte Leistung in den Bereichen Produktion und Hochtechnologie ${ }^{57}$. Die Autoren begründen ihre gleichlautenden Hypothesen zum einen damit, dass der Dienstleistungssektor eine vergleichsweise gröBere Kundennähe aufweist. Eine diverse Belegschaft spiegelt die Heterogenität der Kundschaft wider, was für das Unternehmen Vorteile am Markt bedeuten kann. Zum anderen können sich die positiven Effekte von Gender Diversity im Dienstleistungsbereich besser entfalten, weil die organisationalen Prozesse hier insgesamt weniger stark formalisiert sind als im Bereich der Produktion und der Hochtechnologie und daher vergleichsweise größere Entscheidungsspielräume bestehen.

Mit einer ähnlichen Argumentation begründen Ali/Kulik/Metz (2009) in ihrer Primärstudie die Hypothese, dass die positiven Effekte von Gender Diversity im Dienstleistungsbereich stärker sind, während die negativen Effekte von Gender Diversity im Produkti-

${ }^{57} \mathrm{Vgl.} \mathrm{Joshi/Roh} \mathrm{(2009).}$ 
onsbereich stärker ausgeprägt sind ${ }^{58}$. Im Sinne des Market-Based-View wird Gender Diversity als eine Ressource verstanden, die den Unternehmen vertiefte Kenntnisse der relevanten Märkte (market insight) verspricht. Eine heterogene Belegschaft spiegelt tendenziell die Heterogenität der potentiellen Kundschaft wider und kann daher genauer auf deren Bedürfnisse eingehen. Im Dienstleistungsbereich wirkt sich Gender Diversity stärker positiv aus als im Produktionsbereich, weil die Nähe zum Markt hier ein relevanterer Erfolgsfaktor ist. Dagegen vermuten die Autoren, dass im Produktionsbereich die in der Self-Categorization- und Social Identity-Theorie vorhergesagten negativen Diversity-Effekte stärker zum Tragen kommen. Sie begründen ihre Vermutung damit, dass hier weniger Interaktionsmöglichkeiten zwischen weiblichen und männlichen Subgruppen bestehen, die positive Erfahrungen mit Mitgliedern der jeweils anderen Subgruppe ermöglichen und so der sozialen Kategorisierung entgegenwirken könnten. In ihrer Studie australischer Archiv-Daten von 155 For-Profit-Organisationen finden die Autoren teilweise Bestätigung für ihre Hypothese.

Zusammenfassend wird damit die Argumentation im Sinne des Marktzugangsparadig$\operatorname{mas}^{59}$ - Diversity spiegelt die Heterogenität potentieller Kunden wider - sowohl bei Joshi/Roh (2009) als auch bei Ali/Kulik/Metz (2009) bestätigt ${ }^{60}$. In ähnlicher Weise lässt sich der nicht explizit begründete Befund des Literatur-Reviews von Kochan et al. (2003) interpretieren, dass Gender Diversity dann positive Effekte auf die Unternehmensperformanz hat, wenn eine kundenorientierte Geschäftsstrategie verfolgt wird, bei der die Nähe zum Markt von elementarer Bedeutung ist ${ }^{61}$. Dagegen fanden Leonard et al. (2004) im Rahmen ihrer empirischen Untersuchung von 700 US-amerikanischen Einzelhandelsgeschäften keine Bestätigung für ihre Hypothese, dass der Umsatz von Einzelhandelsgeschäften steigt, wenn die Kundschaft ebenso geschlechterdivers zusammengesetzt ist wie die jeweilige Belegschaft ${ }^{62}$.

Die Ergebnisse der Metaanalyse von Joshi/Roh (2009) deuten auf einen weiteren Moderationseffekt in dem Sinne hin, dass in Branchen mit einem ausgeglichenen Geschlechterverhältnis der negative Effekt von Gender Diversity auf verschiedene Maße der Organisationsleistung reduziert ist ${ }^{63}$. Erstens ist das Merkmal Gender in einer Branche mit

\footnotetext{
${ }^{58} \mathrm{Vgl}$. Ali/Kulik/Metz (2009).

${ }^{59}$ Vgl. Voigt/Wagner (2007); Klaffke (2009).

${ }^{60} \mathrm{Vgl}$. Ali/Kulik/Metz (2009); Joshi/Roh (2009).

${ }^{61} \mathrm{Vgl}$. Kochan et al. (2003).

${ }^{62} \mathrm{Vgl}$. Leonard et al. (2004).

${ }^{63} \mathrm{Vgl}$. Joshi/Roh (2009).
} 
ausgeglichenem Geschlechterverhältnis weniger salient, weil Gender Diversity im Alltag der Betroffenen weniger auffällt. Unter dieser Bedingung ist die Wahrscheinlichkeit sozialer Kategorisierungsprozesse im Vergleich zu einer Branche mit unausgeglichenem Geschlechterverhältnis reduziert. Darüber hinaus vermuten die Autoren, dass in einem Arbeitskontext mit unausgeglichenem Geschlechterverhältnis generell Stereotype gegenüber unterrepräsentierten Gruppen gepflegt werden, die Prozesse der sozialen Kategorisierung begünstigen. Zusätzlich werden in einem solchen Arbeitskontext Teams, die höhere Anteile unterrepräsentierter Mitglieder (z.B. Frauen, ethnische Minoritäten) aufweisen, tendenziell von Außenstehenden schlechter beurteilt und erhalten weniger Ressourcen als andere Teams, was insgesamt zu geringeren leistungsbezogenen Outcomes führt.

Die Befunde zu den Effekten von Organisationskultur als Moderator sind bislang widersprüchlich: So erwies sich in dem Review von Kochan et al. (2003) eine mitarbeiterorientierte Organisationskultur als verstärkender Moderator der Beziehung zwischen Gender Diversity und Performanz ${ }^{64}$. Dagegen vermuteten Choi/Rainey (2010) in ihrer bereits erwähnten Primärstudie, dass eine ergebnisorientierte Unternehmenskultur (die Leistung und Wettbewerb betont) den Zusammenhang zwischen Gender Diversity und Organisationserfolg reduziert, weil eine solche Kultur nicht zu kooperativem, sondern zu kompetitivem Verhalten motiviert ${ }^{65}$. Sie fanden jedoch keine Bestätigung für diese Hypothese.

\subsubsection{Teameigenschaften}

Joshi/Roh (2009) fanden in ihrer Meta-Analyse einen Moderationseffekt der Teaminterdependenz, verstanden als mehrdimensionales Konstrukt aus Aufgaben-, Ziel- und Ergebnisinterdependenz ${ }^{66}$. Vor dem Hintergrund der Social Categorization-Theorie erwarteten die Autoren, dass eine hohe Teaminterdependenz die gemeinsame Zielausrichtung der Teammitglieder stärkt und daher die negativen Effekte von beziehungsorientierten Diversity-Merkmalen (u.a. Gender) auf die Performanz reduziert werden. Entgegen die-

\footnotetext{
${ }^{64} \mathrm{Vgl}$. Kochan et al. (2003).

${ }^{65}$ Vgl. Choi/Rainey (2010).

${ }^{66} \mathrm{Vgl}$. Joshi/Roh (2009).
} 
ser Erwartung wirken sich die beziehungsorientierten Diversity-Merkmale (u.a. Gender) bei niedriger Team-Interdependenz positiv auf die Leistung aus, bei mittlerer und hoher Teaminterdependenz jedoch negativ. Bei mittlerer Teaminterdependenz ist der Zusammenhang zwischen beziehungsorientierten Diversity-Merkmalen (u.a. Gender) und Performanz am negativsten. Die Autoren erklären diesen Befund wie folgt: Unter der Bedingung mittlerer Teaminterdependenz treten Beeinträchtigungen der Teamarbeit auf, die zwar einerseits das positive Potential der Diversity reduzieren, andererseits jedoch nicht so gravierend sind, dass sich die Teammitglieder zur Zusammenarbeit gezwungen fühlen. Bei niedriger Teaminterdependenz besteht dagegen wenig Grund zur Zusammenarbeit; negative Diversity-Effekte sind daher unwahrscheinlich.

Dieselben Autoren konnten die Annahme bestätigen, dass beziehungsorientierte Diversity (u.a. Gender) mit zunehmender Dauer der Zusammenarbeit im Team einen negativen Einfluss auf die Team-Leistung hat: Der negative Effekt beziehungsorientierter Diversity (u.a. Gender) ist erwartungsgemäß in Teams, die auf langfristige Zusammenarbeit ausgelegt sind (long-term teams), signifikant höher ausgeprägt als in Teams, die auf kurzfristige Zusammenarbeit ausgelegt sind (short-term teams). Die Autoren begründen ihre Hypothese mit der Erwartung, dass die Mitglieder in short-term teams aufgrund des Zeitdrucks über Unterschiede hinwegsehen; in long-term teams würden sich Unterschiede zwischen den Mitgliedern dagegen selbst verstärken, dadurch intensivieren und die Zusammenarbeit behindern. Die gegenteilige Annahme können Choi/Rainey (2010) in ihrer empirischen Primär-Untersuchung an 67 US-amerikanischen Bundesbehörden bestätigen: Bezugnehmend auf die Resource Dependence-Theorie ${ }^{67}$ erwarten die Autoren, dass sich die Zusammenarbeit in heterogenen Teams (bezogen auf ethnische Zugehörigkeit, Geschlecht und Alter) mit zunehmender Bestehensdauer der Teams verbessert, weil der intensivere Kontakt zwischen den Teammitgliedern die negativen Effekte von sozialer Kategorisierung reduziert ${ }^{68}$. Die Autoren liefern jedoch keine Begründung dafür, warum Prozesse der sozialen Kategorisierung im Zeitablauf geringer (anstatt stärker) werden; dennoch konnten sie ihre Hypothese eines positiven Zusammenhangs zwischen Diversity und Performanz in long-term teams für Gender Diversity bestätigen.

\footnotetext{
${ }^{67}$ Vgl. Salancik/Pfeffer (1978).

${ }^{68}$ Vgl. Choi/Rainey (2010).
} 


\subsubsection{Diversity-Management}

Kochan et al. (2003) fanden in ihrem Literatur-Review Hinweise auf einen moderierenden Effekt sogenannter ,diversity-focused human resource practices “69. Darunter fassen sie personalpolitische Maßnahmen (Rekrutierung, Selektion, Training, Motivation, Anreiz- und Belohnungsstruktur) zusammen, die darauf ausgerichtet sind, die Kompetenzen der Teamleiter und Mitarbeiter im Hinblick auf den Umgang mit Diversity zu erhöhen. Erwartungsgemäß bestand in einer der vier berichteten Studien ein positiver Zusammenhang zwischen Gender Diversity und Performanz, wenn diese Praktiken vorhanden waren. In einer anderen Studie aus diesem Review konnte dagegen ein ähnlicher Moderationseffekt nicht bestätigt werden: Die Autoren dieser Studie erwarteten, dass eine stärkere Teilnahme von Mitarbeitern an speziellen Diversity-Schulungsprogrammen den positiven Zusammenhang zwischen Gender Diversity und Performanz verstärken würde. Entgegen ihrer Hypothese war dieser Zusammenhang im Fall einer hohen Partizipationsrate jedoch negativ; eine Erklärung für diesen Befund liefern die Autoren nicht. Die übrigen in diesem Review berücksichtigten Studien weisen ebenfalls keine signifikanten Zusammenhänge zwischen Gender Diversity und Performanz auf.

Im weiteren Sinne lässt sich auch ein Befund zur Teamführung zum DiversityManagement zählen. Kochan et al. (2003) fanden einen moderierenden Effekt des Geschlechts der jeweiligen Führungskraft ${ }^{70}$ : Während in Teams mit männlichen Führungskräften kein Zusammenhang zwischen Gender Diversity und Performanz bestand, war dieser Zusammenhang in Teams mit weiblichen Führungskräften negativ. Eine theoretische Interpretation dieses Ergebnisses wird von den Autoren nicht vorgenommen.

Zusammenfassend ist festzuhalten, dass damit drei Moderatoren auf der organisationalen Ebene (Beschäftigungssektor, Geschlechterverhältnis in der jeweiligen Branche, Organisationskultur) und zwei Moderatoren auf der Teamebene (Team-Interdependenz und Dauer der Zusammenarbeit im Team) vor dem Hintergrund der vorliegenden Empirie als bestätigt gelten können, während die Befunde zum Diversity-Management als Moderator widersprüchlich sind. Darüber hinaus fällt auf, dass die Theorie der sozialen Kategorisierung bei der Erklärung der Moderationseffekte dominiert: Es werden offensichtlich überwiegend solche Moderatoren untersucht, die in der Praxis Prozesse der sozialen Kategorisierung verhindern können. Dies bestätigt den oben genannten Befund

\footnotetext{
${ }^{69} \mathrm{Vgl}$. Kochan et al. (2003).

${ }^{70}$ Vgl. Kochan et al. (2003).
} 
von Shore et al. (2009), wonach die Perspektive der sozialen Kategorisierung bei der theoretischen Vorhersage der Effekte von Gender Diversity überwiegt. Moderatoren, die im Sinne der Information/Decision-Making-Perspektive die Generierung ausgewogener Entscheidungen begünstigen können, werden dagegen in den hier untersuchten Arbeiten nicht thematisiert. Ein Grund dafür könnte darin liegen, dass die Forschung im Bereich der Gender Diversity die beiden alternativen Theorieansätze zur Vorhersage der Effekte von Gender Diversity als konkurrierend bzw. einander ausschließend betrachtet. ${ }^{71}$ Da Gender zu den unmittelbar sichtbaren Diversity-Merkmalen gehört, die Kategorisierungen begünstigen, ist die Identifikation von solchen Moderatoren, die Prozesse der sozialen Kategorisierung verhindern bzw. entschärfen, nachvollziehbar (Jackson/Joshi/Erhardt, 2003). Auf die Zweckmäßigkeit einer ausschließlichen Fokussierung auf Prozesse der sozialen Kategorisierung im Zusammenhang mit Gender Diversity wird im Rahmen der abschließenden Diskussion eingegangen.

\section{Methodischer Vergleich der ausgewerteten Studien}

Bevor die Ergebnisse im Hinblick auf Implikationen für Forschung und Praxis interpretiert werden, soll an dieser Stelle der Blick auf methodische Aspekte der ausgewerteten Studien gerichtet werden. Dabei werden zunächst die unterschiedlichen Operationalisierungen der unabhängigen und der abhängigen Variablen diskutiert. Im Anschluss werden die Kontexte, die Untersuchungsebenen, die Studiendesigns sowie die Fallzahlen der Studien verglichen und kritisch reflektiert.

\subsection{Gender Diversity}

Die unabhängige Variable Gender Diversity wurde in den vorgestellten Studien unterschiedlich operationalisiert. Eine häufig verwendete Formel zur Messung von Gender Diversity stellt dabei der Heterogenitätsindex von Blau (1977) dar ${ }^{72}$. Darüber hinaus wurden Entropiemaße ${ }^{73}$ und der Heterogenitäts-Index (HI) nach Metzner (2003) verwendet ${ }^{74}$. Schließlich wurde auch auf einfache statistische Kennzahlen wie das arithmetische Mittel oder die Varianz der Geschlechterverteilung, den prozentualen Frauenan-

\footnotetext{
${ }^{71}$ Van Knippenberg et al. (2004)

${ }^{72}$ Vgl. z.B. Hirschfeld et al. (2005); Richard/Ford/Ismail (2006); Ali/Kulik/Metz (2009).

${ }^{73}$ Vgl. Miller/Quingley (1990); Teachman (1980).

${ }^{74}$ Vgl. z.B. Wegge et al. (2008); Joshi/Roh (2009); Choi/Rainey (2010).
} 
teil und Ordinalskalen (männlich dominierte vs. weiblich dominierte vs. ausgeglichene Gruppe) zurückgegriffen ${ }^{75}$. Zum Teil wurden die Untersuchungen durch die Verwendung mehrerer Gender Diversity-Maße abgesichert ${ }^{76}$.

Nach Harrison/Klein (2007) ist die Wahl eines adäquaten Heterogenitätsmaßes von zentraler Bedeutung, weil dieses mit den zugrundeliegenden theoretischen Annahmen korrespondieren sollte ${ }^{77}$. Unterschiede in der Geschlechterzusammensetzung können dabei als Verteilung gegensätzlicher Ansichten über Teamprozesse oder -ergebnisse angesehen werden, die den Teamzusammenhalt schwächen (,diversity as separation“). Ebenfalls plausibel erscheint die Annahme, dass sich Männer und Frauen systematisch hinsichtlich ihrer Wissens- und Erfahrungshintergründe unterscheiden, was sich positiv auf die Gruppenleistung auswirken sollte (,diversity as variety“). Letztlich kann Gender Diversity auch im Sinne von Machtunterschieden zwischen den Geschlechtern interpretiert werden, die wiederum negativ wirken (,diversity as disparity“'). Das ausgewählte Diversity-Maß sollte der zugrundeliegenden theoretischen Annahme entsprechen (z.B. Standardabweichung und mittlere euklidische Distanz für „separation“, Blau- oder Teachman-Index für „,variety“ und Variations- oder Gini-Koeffizient für „disparity“ ${ }^{67}$. Die in dieser Arbeit vorgestellten Studien weisen unter diesem Blickwinkel jedoch nicht immer den geforderten Fit zwischen theoretischer Argumentation und Operationalisierung von Diversity auf. In der uneinheitlichen und teilweise nicht theoriegeleiteten Auswahl der Diversity-Indizes kann daher eine Ursache für die ambivalenten Ergebnisse der Studien liegen ${ }^{79}$. Insbesondere jüngere Studien beziehen sich jedoch auf die von Harrison/Klein (2007) eingebrachte Systematisierung der Diversity-Messung, was auf zukünftig vergleichbarere empirische Ergebnisse hoffen lässt.

\subsection{Organisationserfolg}

Ein ähnlich heterogenes Bild ergibt sich für die abhängige Variable - den Organisationserfolg im weiteren Sinne. Die Outcome-Maße lassen sich danach unterscheiden, ob sie objektiv, das heißt anhand von intersubjektiv nachvollziehbaren, häufig numerisch erfassbaren Kriterien wie der finanziellen Leistung gemessen werden oder ob sie anhand subjektiver, auf individueller Selbst- oder Fremdeinschätzung basierender Skalen

\footnotetext{
${ }^{75}$ Vgl. z.B. Hirschfeld et al. (2005); Carter et al. (2010).

${ }^{76}$ Vgl. z.B. Wegge et al. (2008); Joshi/Roh (2009).

${ }^{77}$ Vgl. Harrison/Klein (2007).

${ }^{78}$ Vgl. Harrison/Klein (2007), S. 1210.

${ }^{79} \mathrm{Vgl}$. Harrison/Klein (2007).
} 
erhoben wurden. So fanden bei den objektiven Maßen etwa finanzielle Kennzahlen (z.B. Verkäufe, Umsatz, Jahresgewinn, Eigenkapitalrendite) ebenso Anwendung wie verschiedene Effektivitäts- und Effizienzmaße (z.B. Zielerreichung, Produktivität der Angestellten, Bearbeitungs- und Reaktionszeiten). Im Unterschied hierzu wurde Leistung u.a. mit Hilfe folgender subjektiver Variablen erfasst: Selbst- oder Fremdeinschätzung der Effektivität und Qualität der geleisteten Arbeit, der Organisationsleistung, der Organisationsressourcen, der Fähigkeiten des Managements und der Kundenzufriedenheit.

Die meisten Studien zogen mehr als ein Leistungsmaß heran; häufig wurden auch gerade subjektive mit objektiven Variablen kombiniert, um im Sinne einer Triangulation mögliche Verzerrungen kontrollieren zu können. Insgesamt zeigt die Übersicht (vgl. Tabelle 1), dass ein breites Spektrum an Maßen für die Leistung einer Organisation herangezogen wurde, über deren Prämissen sich teilweise diskutieren lässt. So ist etwa fraglich, inwieweit sich Leistung am Gehalt eines Mitarbeiters messen lässt oder wie vergleichbar Selbsteinschätzungen sind. Entsprechend kritisierten etwa Pitts/Wise (2010, S. 63) in ihrem Review, dass häufig inadäquate Indikatoren für Leistung verwendet würden, wodurch nur eine indirekte Untersuchung der Beziehung zwischen Diversity und Organisationserfolg stattfinde ${ }^{80}$. In der Folge sind die vorgestellten Studienergebnisse nur eingeschränkt vergleichbar - werden die Ergebnisse aufeinander bezogen, so ist das jeweilige Verständnis von Organisationserfolg zu spezifizieren.

4.3 Untersuchungskontexte, Art der Studien, Untersuchungsebenen und Stichproben Im Hinblick auf die Kontexte bzw. Branchen, in denen die Studien durchgeführt wurden, dominiert der privatwirtschaftliche Sektor. Die untersuchten Unternehmen gehören u.a. dem Finanzsektor ${ }^{81}$, dem Einzelhandel ${ }^{82}$, der Gastronomie ${ }^{83}$ und der Informationsverarbeitungsbranche ${ }^{84}$ an. Aber auch im öffentlichen Sektor wurden mehrere Studien durchgeführt, so unter anderem in der öffentlichen Verwaltung ${ }^{85}$, in militärischen Kontexten $^{86}$ und an einer Universität ${ }^{87}$.

\footnotetext{
${ }^{80}$ Vgl. Pitts/Wise (2010), S. 63.

${ }^{81} \mathrm{Vgl}$. z.B. Richard/Ford/Ismail (2006).

${ }^{82}$ Vgl. z.B. Kochan et al. (2003); Leonard et al. (2004).

${ }^{83}$ Vgl. z.B. Gonzalez/Denisi (2009).

${ }^{84}$ Vgl. z.B. Kochan et al. (2003).

${ }^{85}$ Vgl. z.B. Choi (2009); Choi/Rainey (2010).

${ }^{86}$ Vgl. z.B. Hirschfeld et al. (2005).

${ }^{87}$ Vgl. z.B. Shaw (2004).
} 
Diese Beschreibung des Untersuchungsumfeldes zeigt bereits, dass alle Primär-Studien als Feldstudien durchgeführt wurden. Dabei befassen sich die meisten Studien mit Diversity auf Teamebene ${ }^{88}$, eine nicht geringe Zahl mit Diversity auf Organisationsebe$\mathrm{ne}^{89}$ und drei Studien untersuchen Diversity-Effekte auf Individualebene ${ }^{90}$. Teilweise beziehen sich die Untersuchungen auch auf mehrere Ebenen ${ }^{91}$. Sowohl die Dominanz von Feldstudien ${ }^{92}$ als auch die Präferenz für die Teamebene ist typisch für die gesamte Diversity-Forschung ${ }^{93}$. Dabei fällt auf, dass in den einzelnen Studien sowie in der MetaAnalyse und den Literatur-Reviews sehr unterschiedliche Arten von Teams untersucht wurden, so zum Beispiel Top Management Teams oder Aufsichtsräte ${ }^{94}$, studentische Projektteams ${ }^{95}$ und Teams in Restaurants ${ }^{96}$. Schließlich variierte die Stichprobengröße erheblich: Die Anzahl der untersuchten Teams bzw. Organisationen bewegt sich zwischen $26^{97}$ und $700^{98}$.

Letztlich sei auf Einschränkungen bei der Ergebnisinterpretation metaanalytischer Untersuchungen hingewiesen, die sich aus verschiedenen methodischen Charakteristika ergeben. Als zentraler Kritikpunkt an Metaanalysen wird in der Literatur das so genannte „Äpfel-und-Birnen-Problem“ diskutiert: In einer Meta-Analyse werden mitunter Studien mit sehr unterschiedlichen Fragestellungen, theoretischen Ansätzen oder auch Operationalisierungen miteinander kombiniert, die nur bedingt vergleichbar sind ${ }^{99}$. Darüber hinaus wird oftmals kritisiert, dass in Metaanalysen Studien von unterschiedlicher methodischer Qualität integriert werden und sich methodische Schwächen von Primärstudien auf die Ergebnisse von Metaanalysen übertragen (,garbage-in-garbage-out"Problem $\left.{ }^{100}\right)$. Weitere Probleme der Metaanalyse liegen im Publikationsbias ${ }^{101}$ sowie in der Integration statistisch abhängiger Studienergebnisse ${ }^{102}$. Trotz dieser Schwächen bzw. Einschränkungen werden metaanalytische Studien jedoch von zahlreichen Wissenschaftlern als adäquate Methode angesehen, um einen Überblick über die bestehende

\footnotetext{
${ }^{88}$ Vgl. z.B. Webber/Donahue (2001); Wegge et al. (2008).

${ }^{89}$ Vgl. z.B. Richard/Ford/Ismail (2006); Miller/del Carmen Triana (2009); Choi/Rainey (2010).

${ }^{90}$ Vgl. Choi (2009), Gonzalez/Denisi (2009), Shaw (2004).

${ }^{91}$ Vgl. z.B. Jackson /Joshi/Erhard (2003); Leonard et al. (2004); Pitts/Wise (2010).

92 Vgl. z.B. Kochan et al. (2003).

${ }^{93} \mathrm{Vgl}$. Jackson/May/Whitney (1995).

${ }^{94}$ Vgl. z.B. Miller/del Carmen Triana (2009); Terjesen/Sealy/Singh (2009).

95 Vgl. z.B. Shaw (2004).

${ }^{96}$ Vgl. Gonzales/Denisi (2009).

${ }^{97}$.Vgl. Gonzales/Denisi (2009).

${ }^{98}$ Vgl. Leonard et al. (2004).

99 Vgl. Drinkmann, 1990; Eyseneck, (1978).

${ }^{100} \mathrm{Vgl}$. Borenstein et al. (2009).

${ }^{101}$ Lipsey \& Wilson (2007).

${ }^{102}$ Borenstein et al. (2009).
} 
Forschung zu bekommen. Zu den Vorzügen von Metaanalysen zählen nicht zuletzt die Systematik ihrer Vorgehensweise, ihre im Vergleich zu narrativen Reviews größere intersubjektive Nachvollziehbarkeit und die Quantifizierung zentraler Tendenzen von Effektstärken ${ }^{103}$.

Zusammenfassend offenbart der Vergleich der Studien eine beachtliche methodische „Diversity“. Verallgemeinernde Aussagen über die Ergebnisse der sehr unterschiedlichen Studien sind daher kaum möglich. Die Forschungsbeiträge decken eine große Bandbreite von Operationalisierungen, Kontexten und Methoden ab. Daher kann nicht ausgeschlossen werden, dass das zentrale Ergebnis unserer Analyse - die inkonsistenten Befunde über den Zusammenhang zwischen Gender Diversity und Organisationserfolg - unter anderem methodischen Artefakten geschuldet ist.

\section{Diskussion}

\subsection{Zusammenfassung}

Ausgangspunkt unserer Analyse ist die Beobachtung, dass Diversity Management in der Personalpolitik deutscher Unternehmen inzwischen ein prominenter Faktor ist. Dabei wird unter Diversity Management einerseits die gezielte Förderung von Diversity und andererseits der Umgang mit einer aufgrund gesellschaftlicher Wandlungsprozesse immer heterogener werdenden Belegschaft zusammengefasst. Im Zentrum einschlägiger Konzepte des Diversity Management steht dabei oftmals Gender Diversity. Neben der Reaktion auf die politisch-normative Erwünschtheit bzw. Notwendigkeit, dem Gleichstellungsgedanken Rechnung zu tragen, herrscht in vielen Organisationen die Überzeugung vor, dass Gender Diversity auch einen ökonomischen Erfolgsfaktor darstellt ${ }^{104}$.

Vor diesem Hintergrund diskutiert der vorliegende Beitrag aktuelle empirische Befunde zum Zusammenhang zwischen Gender Diversity und organisationaler Performanz. Die Ergebnisse der hier referierten empirischen Studien zeigen erstens, dass die direkten Effekte von Gender Diversity auf den Organisationserfolg widersprüchlich sind. Ein klarer Trend, welche Effekte überwiegen, lässt sich daher nicht identifizieren. Diese Inkonsistenz der Ergebnisse lässt sich teilweise anhand der ebenfalls widersprüchlichen

${ }^{103}$ Vgl. z.B. Cooper (2010); Lipsey \& Wilson (2007); Rustenbach (2003).

${ }^{104}$ Vgl. Singh/Point (2006). 
theoretischen Erwartungen bezüglich der Effekte von Gender Diversity erklären. So werden im Sinne der Information/Decision-Making-Perspektive ${ }^{105}$ positive Effekte von Gender Diversity erwartet, während die Social Categorization-Perspektive ${ }^{106}$ negative Effekte vorhersieht.

Entsprechend wurden im zweiten Schritt eine Reihe von Studien identifiziert, die kritische Randbedingungen (Moderatoren) des Zusammenhangs zwischen Gender Diversity und Organisationserfolg untersuchen. Dabei handelt es sich um eine große Bandbreite von Faktoren, von denen viele jedoch bisher nur in Einzelstudien identifiziert wurden und daher kaum verallgemeinert werden können (vgl. Anhang). Betrachtet man nur diejenigen Moderatoren, die in Meta-Analysen und/oder Literatur-Reviews untersucht wurden, so bestätigt die vorliegende Empirie organisationale Rahmenbedingungen der Teamarbeit (Beschäftigungssektor, Grad an Gender Diversity in der jeweiligen Branche und Organisationskultur) und Eigenschaften des Teams (Grad der Teaminterdependenz und Dauer der Zusammenarbeit) als moderierende Randbedingungen. Bei der Interpretation dieser Ergebnisse ist allerdings einschränkend darauf hinzuweisen, dass die identifizierten Arbeiten zu den Effekten von Gender Diversity in methodischer Hinsicht sehr heterogen sind, was die Generalisierbarkeit der Ergebnisse zusätzlich erschwert.

\subsection{Implikationen für die Forschung}

Die Forschung über den Zusammenhang zwischen Gender Diversity und Organisationserfolg erweist sich in konzeptioneller und methodischer Hinsicht als unausgereift. Auch aus diesem Grund lassen sich die bisherigen Ergebnisse kaum sinnvoll zusammenfassen. Im Folgenden werden daher Ansätze diskutiert, die für die künftige Forschung zu Gender Diversity von Bedeutung sein könnten.

In konzeptioneller Hinsicht wurden neben den dargestellten direkten Effekten und Moderationseffekten auch gelegentlich Mediator-Variablen untersucht, die den jeweiligen Zusammenhang erklären. Beispielsweise fanden Kochan et al. (2003) einen Mediationseffekt konstruktiver Gruppenprozesse, die sich fördernd auf die Leistung geschlechterheterogener Teams auswirkten ${ }^{107}$. Dagegen konnten Miller/del Carmen Tria-

\footnotetext{
105 Vgl. van Knippenberg/Schippers (2007)

106 Vgl. van Knippenberg/Schippers (2007)

107 Vgl. Kochan et al. (2003), Vol. 42, S. 3-21.
} 
$n a$ (2009) die angenommenen Mediatoren „Firmenreputation“ und „Innovativität“ des Zusammenhangs zwischen Gender Diversity und Unternehmensperformanz in rund 300 „Fortune 500“-Unternehmen nicht nachweisen ${ }^{108}$. Eine stärkere Konzentration auf die Untersuchung mediierender Prozesse fehlt jedoch bislang ebenso wie ein gemeinsames theoretisches Bezugssystem für die Analyse von Mediations- und Moderationseffekten. Dies erscheint umso gravierender als in der erfolgreichen Identifikation mediierender Prozesse zwischen Gender Diversity und Organisationserfolg der Schlüssel für eine präzise Analyse moderierender Variablen liegt ${ }^{109}$.

Augenfällig ist zudem die einseitige Konzentration auf negative Effekte von Gender Diversity im Sinne der Social Categorization-Perspektive, die sich auch in der Auswahl der untersuchten Moderatoren widerspiegelt. In einer kritischen Analyse der gesamten Diversity-Forschung konstatierten van Knippenberg et al. (2004) bereits vor einigen Jahren, dass eine einseitige Konzentration auf positive oder negative Effekte bestimmter Diversity-Merkmale angesichts der Heterogenität der empirischen Befundlage nicht angebracht sei. Vielmehr müsse davon ausgegangen werden, dass jedes DiversityMerkmal (so auch Gender Diversity) potentiell sowohl positive als auch negative Effekte haben kann. Um das Zusammenspiel positiver und negativer Effekte von Diversity auf die Performanz systematisch zu erschließen, integrieren die Autoren die konkurrierenden theoretischen Ansätze in ein einheitliches theoretisches Modell, das so genannte „Categorization Elaboration Model“"110.

Orientiert man sich an diesem Modell, würde es sich etwa anbieten, als Mediator zwischen Gender Diversity und Performanz die Verarbeitung aufgabenrelevanter Informationen in den Vordergrund zu stellen, die im Sinne der Information/Decision-MakingPerspektive einen positiven Einfluss auf organisationale Performanz ausübt. Darauf aufbauend kann systematisch nach Moderatoren gesucht werden, die den Zusammenhang zwischen Gender Diversity und diesem Mediator beeinflussen. Das Categorization-Elaboration-Modell nennt hier neben Prozessen der sozialen Kategorisierung Aspekte wie Konflikte, Kohäsion oder Commitment. Zugleich postuliert das Modell die Identifikation von Moderatoren, die die positiven (Gender) Diversity-Effekte in Form verbesserter Informationsverarbeitung begünstigen, wie etwa aufgabenbezogene Motivation und Fähigkeiten der Teammitglieder. Eine stärkere Konzentration auf solche Mode-

\footnotetext{
108 Vgl. Miller/del Carmen Triana (2009).

109 Vgl. van Knippenberg/Schippers (2007).

${ }^{110}$ van Knippenberg et al. (2004).
} 
ratoren, die die positiven Effekte von Gender Diversity begünstigen, erscheint angesichts der Einseitigkeit der bisher untersuchten Moderatoren als wünschenswert. Darüber hinaus ist das Merkmal Gender vergleichsweise stark ideologisch besetzt, was die Salienz von Gender Diversity erhöht und damit Prozesse der sozialen Kategorisierung tendenziell beschleunigt. Hierbei dürfte insbesondere der sogenannte „,normative fit““ (van Knippenberg et al., 2004) eine Rolle spielen, definiert als das Ausmaß, in dem das Merkmal Geschlecht von den Teammitgliedern als aufgabenrelevant wahrgenommen wird. Vor diesem Hintergrund wären z.B. Annahmen darüber interessant, welches Verhältnis von Frauen/Männern in einem Team bei welcher Machtverteilung für welche Art von Aufgaben erfolgversprechend ist.

Eine Orientierung der Forschung zu Gender Diversity an einem solchen oder ähnlichen theoretischen Bezugsrahmen würde es künftig erleichtern, Untersuchungsergebnisse vergleichbar zu machen, widersprüchliche Ergebnisse aufzuklären und generalisierbare Aussagen über die Effekte von Gender Diversity zu erzielen. Auf dieser Basis wäre es darüber hinaus möglich, Ansatzpunkte für ein Diversity Management zu entwickeln, das die identifizierten Moderatoren des Zusammenhangs zwischen Gender Diversity und Performanz in der erwünschten Weise beeinflusst.

Bei der Entwicklung eines konsistenten theoretischen Bezugsrahmens wäre darüber hinaus zu überlegen, inwieweit die Fokussierung auf ökonomische Performanz überwunden werden kann. Die unterschiedlichen Motive für Diversity Management sowie die wenig konsistenten Ergebnisse unserer Analyse zum ökonomischen Erfolg von Gender Diversity deuten darauf hin, dass es sinnvoll sein könnte, verstärkt andere Outcomes als abhängige Variablen zu untersuchen. In Frage kämen etwa die gesellschaftliche Resonanz auf Gender Diversity, die Reputation der Unternehmen oder Zufriedenheit, Gerechtigkeitsempfinden und „well-being“ der Mitarbeiter.

In methodischer Hinsicht erschwert die Vielfalt an Operationalisierungen, Untersuchungskontexten und verwendeten Moderator-, Mediator- und Outcome-Variablen die Vergleichbarkeit der Forschungsergebnisse und behindert damit den Erkenntnisfortschritt zu den Effekten von Gender Diversity. Analog zur Entwicklung eines einheitlichen konzeptionellen Referenzrahmens erscheint daher eine methodische Konvergenz in der Gender Diversity-Forschung bis zu einem gewissen Grad als sinnvoll, ohne natürlich die Koexistenz verschiedener Forschungsparadigmen in Frage zu stellen. Künftige 
Untersuchungen könnten dabei insbesondere im Hinblick auf die folgenden beiden Aspekte einen Beitrag zur Vergleichbarkeit von Forschungsergebnissen leisten. Erstens sollte die Operationalisierung von Gender Diversity mit der zugrundeliegenden theoretischen Argumentation korrespondieren; je nachdem, ob Gender Diversity primär als Potential für einen erweiterten kognitiven Ressourcenpool oder als Risiko für Gruppenprozesse und Teamkohäsion gesehen wird, sollte das entsprechende Diversity-Maß gewählt werden ${ }^{111}$. Zweitens sollte die Forschung zu den Effekten von Gender Diversity stärker in „echten“ organisationalen Settings durchgeführt werden. So interessant Studien im Militärbereich oder im universitären Umfeld auch sein mögen: Die Generalisierbarkeit ihrer Ergebnisse auf privatwirtschaftliche Organisationen und öffentliche Verwaltungen dürfte eher begrenzt sein.

Darüber hinaus lag der Fokus der Forschung über Gender Diversity bisher weit überwiegend auf Organisationen in den USA. Es ist anzunehmen, dass gerade für die Auswirkungen von Gender Diversity und Diversity Management nicht nur der organisationale, sondern auch der gesellschaftliche Kontext eine entscheidende Rolle spielt ${ }^{112}$. Internationale bzw. interkulturelle Vergleichsstudien über die Effekte von Gender Diversity liegen jedoch bislang nicht vor. Daher sind die Ergebnisse der vorliegenden Empirie nur mit Vorsicht auf den deutschsprachigen Raum zu übertragen, wo der Grad an Gender Diversity noch vergleichsweise gering ausfällt ${ }^{113}$. Grundlagen für Empfehlungen an die Praxis deutscher Unternehmen können daher nur aus einer verstärkten Forschungstätigkeit im deutschsprachigen Kontext gewonnen werden.

\subsection{Implikationen für die Praxis}

Das entscheidende Ergebnis der vorliegenden Analyse für die Unternehmenspraxis dürfte in der Tatsache liegen, dass Gender Diversity nicht von sich aus, gleichsam ,automatisch“, die Leistung eines Teams oder einer Organisation steigert. Optimistische Erwartungen an die ökonomische Effizienz von Gender Diversity sind daher durch die bestehende empirische Forschung in keiner Weise legitimiert. Ob und in welchem Maße das in Gender Diversity theoretisch vermutete Potential beispielsweise im Sinne der Information/Decision-Making-Perspektive ${ }^{114}$ in der Praxis realisiert werden kann, scheint daher von den jeweiligen Randbedingungen abzuhängen. Angesichts der oben

\footnotetext{
$111 \mathrm{Vgl}$. Harrison/Klein (2007).

112 Vgl. Krell (2009); Jackson/Joshi (2011).

${ }^{113} \mathrm{Vgl}$. Dolado/Felgueroso/Jimeno (2002).

${ }^{114}$ Vgl. u.a. Knippenberg/Schippers (2007).
} 
geschilderten Inkonsistenz und Fragmentiertheit der bisherigen empirischen Forschung zu diesen Randbedingungen wäre es jedoch verfrüht, konkrete Schlüsse für die organisationale Praxis zu ziehen.

Versucht man dennoch die Frage zu beantworten, in welchen Kontexten Gender Diversity zu positiven bzw. negativen Effekten führt, ergeben sich aus der vorliegenden Untersuchung die folgenden Hinweise: Eine entscheidende Rolle für die ökonomischen Effekte von Gender Diversity scheint erstens die Branche zu spielen, in der sich die jeweilige Organisation befindet. So spricht einiges dafür, positive Effekte eher im Dienstleistungsbereich zu erwarten, während im Produktions- und HochtechnologieBereich tendenziell mit negativen Effekten zu rechnen ist ${ }^{115}$. Ähnlich ist der Befund einzuschätzen, dass positive Effekte eher in solchen Branchen zu erwarten sind, in denen das Geschlechterverhältnis bereits ausgewogen ist ${ }^{116}$. Praktiker sollten daher bei der Abwägung der Frage, ob Gender Diversity auch ökonomische Vorteile mit sich bringt, in erster Linie die Besonderheiten ihrer jeweiligen Branche berücksichtigen. Wie die Studie von Kochan et al. (2003) zeigt, kann die Etablierung einer ,,mitarbeiterorientierten“ Organisationskultur dazu beitragen, die Chance positiver Diversity-Effekte zu erhöhen. Nimmt man an, dass Organisationskulturen zwar von Branchenkulturen beeinflusst, aber nicht determiniert werden, wird hier ein Gestaltungsspielraum für die Praxis erkennbar. Zweitens kann dagegen die Annahme, dass die Bilanz aus positiven und negativen Diversity-Effekten durch eine angemessene Arbeitsgestaltung verbessert werden kann, bisher nicht bestätigt werden. So nehmen positive Diversity-Effekte nicht wie erwartet durch eine Erhöhung der Teaminterdependenz $\mathrm{zu}^{117}$. Praktiker sollten die mit Gender Diversity verbundenen Risiken daher nicht vorschnell für leicht beherrschbar halten.

Zusammenfassend erscheint es damit ausgesprochen problematisch, die Förderung von Gender Diversity primär ökonomisch zu legitimieren. Eine ökonomische Legitimation kann sogar kontraproduktiv wirken, wenn im Ergebnis nicht nur die erwarteten positiven Diversity-Effekte ausbleiben, sondern sich spürbare negative Effekte einstellen, die das Konzept der Gender Diversity als solches diskreditieren. Beim derzeitigen Stand der Forschung lässt sich die gezielte Förderung von Gender Diversity daher ausschließlich

\footnotetext{
115 Joshi/Roh (2009).

116 Joshi/Roh (2009).

117 Joshi/Roh (2009).
} 
normativ legitimieren ${ }^{118}$. Von einer Forschung, die solche Gender Diversity-Effekte untersucht, die über die ökonomische Performanz hinausgehen (vgl. oben), würde die Praxis daher unmittelbar profitieren.

Abschließend soll an dieser Stelle überlegt werden, welche Maßnahmen eines Diversity Managements aus theoretischer Sicht sinnvoll sein können. Um die im Sinne der Information/Decision-Making Perspektive erwarteten positiven Effekte gemischtgeschlechtlicher Teams zu realisieren, erscheinen auf den ersten Blick solche Maßnahmen als sinnvoll, die es erlauben, mögliche geschlechtsspezifische Erfahrungshintergründe oder Stärken der Teammitglieder nicht nur zu tolerieren, sondern gezielt für die anstehenden Aufgaben einzusetzen. Wie unsere Studie zeigt, konnten entsprechende Effekte jedoch bisher weder für sogenannte Diversity-bezogene Human Resource-Praktiken noch für Diversity-bezogene Schulungsmaßnahmen konsistent nachgewiesen werden (Kochan et al. 2003).

Die folgenden Überlegungen setzen daher alternativ an der Frage an, wie die im Sinne der Social-Categorization-Perspektive ${ }^{119}$ zu erwartenden negativen Effekte von Gender Diversity reduziert werden können. Wenn dysfunktionale soziale Kategorisierungen vor allem auf die Bildung von Subgruppen zurückzuführen sind, die anschließend in Konflikt(e) miteinander geraten, läge ein erster Ansatzpunkt darin, die Bildung von Subgruppen in geschlechterdiversen Teams entweder zu verhindern oder ihre konfliktinduzierende Wirkung zu unterbinden. Für beide Ziele wird in der Literatur die Etablierung einer sogenannten gemeinsamen sozialen Identität diskutiert ${ }^{120}$. Eine gemeinsame, auf das jeweilige Team oder die jeweilige Organisation bezogene soziale Identität aller Gruppenmitglieder würde dazu beitragen, dass geschlechterbezogene Identitäten („Wir Männer“ bzw. „Wir Frauen“) weniger salient sind und damit die Bildung von Subgruppen weniger wahrscheinlich wird. Eine gemeinsame soziale Identität könnte darüber hinaus dazu beitragen, dass selbst im Fall der Existenz von Subgruppen die Konfliktwahrscheinlichkeit abnimmt: Unter der Voraussetzung eines gemeinsamen Ziels werden Auffassungsunterschiede zwischen Subgruppen (hier: Männern vs. Frauen) weniger im Sinne persönlicher Bedrohungen interpretiert und führen daher weniger stark zu Spannungen.

\footnotetext{
118 Vgl. Krell (2009); Carter et al. (2010).

${ }^{119} \mathrm{Vgl}$. Tajfel (1981).

${ }^{120}$ Vgl. Hogg/Abrams (1988); Hogg/Terry (2001); van Knippenberg/Ellemers (2003).
} 
Hinsichtlich der Frage, wie eine gemeinsame Identität in geschlechterdiversen Teams geschaffen werden kann, rückt unter anderem die Führung von Teams in den Vordergrund. Führungskräfte könnten über die Betonung von Gemeinsamkeiten und die Formulierung teambezogener Ziele eine gemeinsame Identität des Teams betonen. Darüber hinaus könnten Führungskräfte aber auch gezielt über Chancen und Gefahren von Gender Diversity aufklären, um einen konstruktiveren Umgang mit Gender Diversity zu erreichen. Die Untersuchung von Führung als Moderator der Diversity-PerformanzBeziehung stellt sowohl in der Gender Diversity-Forschung ${ }^{121}$ als auch in der allgemeinen Diversity-Forschung ${ }^{122}$ eine vielversprechende aktuelle Entwicklung dar ${ }^{123}$.

\footnotetext{
${ }^{121}$ Vgl. Shore et al. (2009).

122 Vgl. z.B. Hüttermann/Boerner (2011).

${ }^{123}$ Vgl. Jackson/Joshi/Erhardt (2003).
} 


\section{Literaturverzeichnis}

Ali, Muhammad/Kulik, Carol T./Metz, Isabel (2009), The Impact of Gender Diversity on Performance in Service and Manufacturing Organizations, University of Melbourne, Melbourne.

Allianz (2011), Warum ist Diversity bei der Allianz ein wichtiges Thema?, https://www.allianz.com/de/karriere/informationen-fuerbewerber/faq/diversity/diversity.html (17.09.2011).

Bundesagentur für Arbeit (2011), Perspektive 2025: Fachkräfte für Deutschland, http://www.arbeitsagentur.de/zentralerContent/Veroeffentlichungen/Sonstiges/Perspektive-2025.pdf (17.09.2011).

Berscheid, Ellen/Walster, Elaine H. (1978), Interpersonal Attraction, Menlo Park.

Blau, Peter Michael (1977), Inequality and heterogeneity. A primitive theory of social structure, New York.

Borenstein, Michael/Hedges, Larry V./Higgins, Julian. P. T./Rothstein, Hannah R. (2009), Introduction to meta-analysis, London.

Bowers, Clint A./Pharmer, James. A./Salas, Eduardo (2000), When Member Homogeneity is Needed in Work Teams, in: Small Group Research, Vol. 3, S. 305-327.

Bratter, Jenifer/Zuberi, Tukufu (2001), The Demography of Difference: Shifting Trends of Racial Diversity and Interracial Marriage 1960-1990, in: Race and Society, Vol 4, S. 133-148.

Brewer, Marilynn B. (1979), In-group bias in the minimal intergroup situation: A cognitive-motivational analysis, in: Psychological Bulletin,Vol. 86, S. 307-324.

Deutscher Bundestag (2011), Schröder verteidigt Flexi-Quote gegen Kritik der Oppositionsfraktionen, http://www.bundestag.de/presse/hib/2011_05/2011_193/01.html (13.09.2011).

Byrne, Donn (1971), The Attraction paradigm, New York.

Carter, David, A./D'Zousa, Frank/Simkins, Betty, J./Simpson, W. Gary (2010), The Gender and Ethnic Diversity of US Boards and Board Committees and Firm Financial Performance, in: Corporate Governance: An International Review, Vol. 18, S. 396-414.

Certo, Trevis S. (2003), Influencing initial public offering investors with prestige: Signaling with board structures, in: Academy of Management Review, Vol. 28, S. $432-446$. 
Choi, Sungjoo (2009), Diversity in the US Federal Government: Diversity Management and Employee Turnover in Federal Agencies, in: Journal of Public Administration Research and Theory, Vol. 19, S. 603-630.

Choi, Sungjoo/Rainey, Hal G. (2010), Managing Diversity in U.S. Federal Agencies: Effects of Diversity and Diversity Management on Employee Perceptions of Organizational Performance, in: Public Administration Review, Vol. 70, S. 109121.

Cooper, Harris M. (2010), Research synthesis and meta-analysis: A step-by-step approach, Thousand Oaks.

Cyert, Richard M./March, James G. (1963), A behavioral theory of the firm, Englewood Cliffs.

Deckstein, Dagmar (2008), Talente: Ursula Schwarzenbart. Die Männer-Versteherin, in: Süddeutsche Zeitung, 06. Oktober 2008, S. 23.

Deutsche Bahn (2010), Why we support diversity. Individuality as a guarantee for DB's success,

http://www.deutschebahn.com/site/bahn/en/career/diversity/diversity.html (17.09.2011).

Dolado, Juan J./Felgueroso, Florentino/Jimeno, Juan F. (2002), Recent Trends in Occupational Segregation by Gender: A Look Across the Atlantic. Forschungsinstitut zur Zukunft der Arbeit Discussion Paper Vol. 524, Bonn.

Drinkmann, A. (1990), Methodenkritische Untersuchungen zur Metaanalyse, Weinheim.

Dwyer, Sean/Richard, Orlando C./Chadwick, Ken (2003), Gender diversity in management and firm performance: the influence of growth orientation and organizational culture, in: Journal of Business Research, Vol. 56, S. 11.

Gonzalez, Jorge A./Denisi, Angelo S. (2009), Cross-level effects of demography and diversity climate on organizational attachment and firm effectiveness, in: Journal of Organizational Behavior, Vol. 30, S. 21-40.

Harrison, David A./Klein, Katherine J. (2007), What's the Difference? Diversity Constructs as Separation, Variety, or Disparity in Organizations, in: Academy of Management Review, Vol. 32, S. 1199-1228.

Herring, Cedric (2009), Does Diversity Pay? Race, Gender, and the Business Case for Diversity, in: American Sociological Review, Vol. 74, S. 208-224.

Hirschfeld, Robert R./Jordan, Mark H./Feild, Hubert S./Giles, William F./Armenakis, Achilles A. (2005), Teams' female representation and perceived potency as 
inputs to team outsomes in a predominantly male field setting, in: Personnel Psychology, Vol. 58, S. 893-924.

HMTH - Hochschule für Musik und Theater Hannover (2010), Stellenausschreibungen: Volontariat im Bereich des künstlerisch-wissenschaftlichen Projektmanagement, http://www.hmtm-hannover.de/de/hochschule/stellenausschreibungen (16.09.2010).

Hogg, Michael A./Abrams, Dominic (1988), Social identifications - A social psychology of intergroup relations and group processes, London.

Hogg, Michael A./Terry, Deborah J. (2001), Social identity theory and organizational processes, in: Hogg, Michael A./Terry, Deborah J. (Hrsg.), Social identity processes in organizational contexts, Philadelphia, S. 1-12.

Hüttermann, Hendrik/Boerner, Sabine (i2011), Fostering innovation in functionally diverse teams: The two faces of transformational leadership, in: European Journal of Work and Organizational Psychology, Vol. 20, S. 833-854.

Jackson, Susan. E./Joshi, Aparna (2011), Work Team Diversity. In S. Zedeck (Hrsg.), APA Handbook of Industrial and Organizational Psychology, Washington D.C., Vol. 1, S. 651-686.

Jackson, Susan E./Joshi, Aparna/Erhardt, Niclas L. (2003), Recent Research on Team and Organizational Diversity: SWOT Analysis and Implications, in: Journal of Management, Vol. 29, S. 801-830.

Jackson, Susan E./May, Karen E./Whitney, Kristina (1995), Understanding the Dynamics of Diversity in Decision-Making Teams, in: Guzzo, Richard A./Salas, Eduardo (Hrsg.), Team effectiveness and decision making in organizations, San Francisco, S. 204-261.

Joshi, Aparna/Roh, Hyuntak (2009), The role of context in work team diversity research: A meta-analytical review, in: Academy of Management Journal, Vol. 52, S. 599-627.

Klaffke, Martin (2009), Wandel durch Diversity Management. Mitarbeitervielfalt als Erfolgspotenzial strategisch nutzen, in: Klaffke, Martin (Hrsg.), Strategisches Management von Personalrisiken, Wiesbaden, S. 139-158.

Kochan, Thomas/Bezrukova, Katerina/Ely, Robin/Jackson, Susan/Joshi, Aparna/Jehn, Karen/Leonard, Jonathan/Levine, David/Thomas, David (2003), The effects of diversity on business performance: Report of the diversity research network, in: Human Resource Management,Vol. 42, S. 3-21. 
Köppel, Petra (2010), Diversity Management in Deutschland - eine Unternehmensbefragung, in: Badura, Bernhard/Schröder, Helmut/Klose, Joachim/Macco, Katrin (Hrsg.), Fehlzeiten-Report 2010. Vielfalt managen: Gesundheit fördern - Potenziale nutzen, Berlin, S. 23-35.

Krell, Gertraude (2009), Gender und Diversity: Eine ,Vernunftehe“ - Plädoyer für vielfältige Verbindungen, in: Andresen, Sünne/Koreuber, Mechthild/Lüdke, Dorothea (Hrsg.), Gender und Diversity: Albtraum oder Traumpaar?, Wiesbaden, S. 133-153.

Krell, Gertraude/ Ortlieb, Renate/Sieben, Barbara (Hrsg.) (2011). Chancengleichheit durch Personalpolitik. Gleichstellung von Frauen und Männern in Unternehmen und Verwaltungen. Rechtliche Regelungen - Problemanalysen - Lösungen. 6., vollst. überarb. u. erw. Auflage. Wiesbaden: Gabler.

Leonard, Jonathan S./Levine, David I./Joshi, Aparna (2004), Do birds of a feather shop together? The effects on performance of employees' similarity with one another and with customers, in: Journal of Organizational Behavior, Vol. 25, S. 731-754.

Lipsey, Mark W./Wilson, David B. (2007), Practical meta-analysis, Thousand Oaks.

Metzner, Cornelia (2003), Eine Analyse des Konzepts der Homogenitäts- und Heterogenitätsbalancierung in der Zusammensetzung von Arbeitsgruppen, unveröffentlichtes Manuskript, Universität Regensburg, Regensburg.

Miller, Toyah/del Carmen Triana, María (2009), Demographic Diversity in the Boardroom: Mediators of the Board Diversity-Firm Performance Relationship, in: Journal of Management Studies, Vol. 46, S. 755-786.

Miller, Vincent. P./Quigley, John M. (1990), Segregation by Racial and Demographic Group: Evidence from the San Francisco Bay Area, in: Urban Studies, Vol. 27, S. 3-21.

Pitts, David. W./Wise, Lois R. (2010), Workforce Diversity in the New Millennium: Prospects for Research, in: Review of Public Personnel Administration, Vol. 30, S. 44-69.

Powell, Walter W./DiMaggio, Paul J. (1991), The New Institutionalism in Organizational Analysis, Chicago.

Richard, Orlando C./Ford, David/Ismail, Kiran (2006), Exploring the performance effects of visible attribute diversity: the moderating role of span of control and organizational life cycle, in: International Journal of Human Resource Management, Vol. 17, S. 2091-2109. 
Richard, Orlando. C./Barnett, Tim/Dwyer, Sean/Chadwick, Ken (2004), Cultural diversity in management, firm performance, and the moderating role of entrepreneurial orientation dimensions, in: Academy of Management Journal, Vol. 47, S. 255-266.

Rustenbach, Stephan Jeff (2003), Metaanalyse: Eine anwendungsorientierte Einführung, Bern.

Salancik, Gerald R./Pfeffer, Jeffrey (1978), The External control of organizations: a resource dependence perspective, New York.

SAP (2011), Unsere Mitarbeiter, http://www.sap.com/corporate-de/our-company/ourpeople.epx (17.09.2011).

Shapcott, Kim M./Carron, Albert V./Burke, Shauna M./Bradshaw, Michael H./ Estabrooks, Paul A. (2006), Member Diversity and Cohesion and Performance in Walking Groups in: Small Group Research, Vol. 37, S. 701-720.

Shaw, James B. (2004), A Fair Go for All? The Impact of Intragroup Diversity and Diversity-Management Skills on Student Experiences and Outcomes in TeamBased Class Projects, in: Journal of Management Education, Vol. 28, S. 139169.

Shore, Lynn M./Chung-Herrera, Beth G./Dean, Michelle A./Ehrhart, Karen H./Jung, Don I./Randel, Amy E./Singh, Gangaram (2009), Diversity in organizations: Where are we now and where are we going?, in: Human Resource Management Review, Vol. 19, S. 117-133.

Singh, Val/Point, Sébastien (2006), (Re)Presentations of Gender and Ethnicity in Diversity Statements on European Company Websites, in: Journal of Business Ethics, Vol. 68, S. 363-379.

Süß, Stefan (2009), Die Institutionalisierung von Managementkonzepten. Eine strukturationstheoretisch-mikropolitische Perspektive, in: Zeitschrift für Betriebswirtschaft, 79. Jg., S. 187-212.

Süß, Stefan (2008), Diversity-Management auf dem Vormarsch. Eine empirische Analyse der deutschen Unternehmenspraxis, in: ZfbF, 60. Jg., S. 406-430.

Tajfel, Henri (1981), Human groups and social categories - Studies in social psychology, Cambridge.

Tajfel, Henri/Turner, John C. (1986), Social identity theory and intergroup behavior, in: Worchel, Stephen/ Austin, William G. (Hrsg.), Psychology of intergroup relations, Chicago, S. 7-24. 
Teachman, Jay D. (1980), Analysis of population diversity, in: Sociological Methods and Research, Vol. 8, S. 341-362.

Telekom, (2010), Deutsche Telekom führt als erstes Dax-30-Unternehmen Frauenquote für die Führung ein, http://www.telekom.com/dtag/cms/content/dt/de/829454 (13.09.2011).

Terjesen, Siri/Sealy, Ruth/Singh, Val (2009), Women Directors on Corporate Boards: A Review and Research Agenda, in: Corporate Governance: An International Review, Vol. 17, S. 320-337.

Turner, John C./Hogg, Michael/Oakes, Penelope J./Reicher, Stephen D./Wetherell, Margaret S. (1987), Rediscovering the social group: a self-categorization theory, Oxford.

van Knippenberg, Daan/De Dreu, Carsten K. W./Homan, Astrid C. (2004), Work group diversity and group performance: An integrative model and research agenda, in: Journal of Applied Psychology, Vol. 89, S. 1008-1022.

van Knippenberg, Daan/Ellemers, Naomi (2003), Social identity and group performance: Identification as a key to collective effort, in: Haslam, S. Alexander/van Knippenberg, Daan/Platow, Michael J./Ellemers, Naomi (Hrsg.), Social identity at work: Developing theory for organizational practice, New York, S. 29-42.

van Knippenberg, Daan/Schippers, Michaéla C. (2007), Work group diversity, in: Annual Review of Psychology, Vol. 58, S. 515-541.

Voigt, Bernd-Friedrich/Wagner, Dieter (2007), Diversity-Management als Leitbild von Personalpolitik, Berlin.

Webber, Sheila S./Donahue, Lisa M. (2001), Impact of highly and less job-related diversity on work group cohesion and performance: a meta-analysis, in: Journal of Management, Vol. 27, S. 141-162.

Wegge, Jürgen/Roth, Carla/Kanfer, Ruth/Neubach, Barbara/Schmidt, Klaus-Helmut (2008), Age and Gender Diversity as Determinants of Performance and Health in a Public Organization: The Role of Task Complexity and Group Size, in: Journal of Applied Psychology, Vol. 93, S. 1301-1313.

Williams, Katherine Y./O'Reilly, Charles A. (1998), Demography and diversity in organizations: A review of 40 years of research, in: Research in Organizational Behavior, Vol. 52, S. 599-627. 


\section{Anhang: Beschreibung der Einzelstudien zu Moderatoren des Zusammenhangs zwischen Gender Diversity und Performance}

\section{Organisationale Einflussfaktoren}

Richard/Ford/Ismail (2006) können für den amerikanischen Bankenbereich $(\mathrm{N}=79)$ bestätigen, dass der Zusammenhang zwischen Gender Diversity und Performanz zum einen in Organisationen mit geringer Kontrollspanne positiver ausfällt als in Organisationen mit großer Kontrollspanne ${ }^{1}$. Sie begründen ihre Hypothese damit, dass Manager bei einer geringen Kontrollspanne eher die Möglichkeit haben, durch koordinierende und integrierende Maßnahmen negativen Effekten von Gender Diversity vorzubeugen. Unter dieser Voraussetzung ist es wahrscheinlicher, dass das aus den verschiedenen Perspektiven der Teammitglieder resultierende breitere Wissen auch tatsächlich implementiert werden kann und damit zur Performanz beiträgt. Die zweite bestätigte Hypothese lautet, dass der Zusammenhang zwischen Gender Diversity und Performanz in Organisationen, die sich in einem frühen Stadium ihres Lebenszyklus befinden, positiver ausfällt als in Organisationen, die sich in einem späten Stadium ihres Lebenszyklus befinden. In frühen Phasen (start up-Phase und Wachstumsphase) müssen Organisationen sich einen Marktzugang erschließen und die Akzeptanz der Kunden erlangen; darüber hinaus stehen in diesem Stadium Forschung und Entwicklung im Vordergrund. Gender Diversity fördert zum einen die Fähigkeit, auf den Bedarf heterogener Kundschaft einzugehen und damit Sensibilität zur Erschließung der Märkte und zum anderen -im Sinne der Information/Decision-Making-Perspektive- die Kreativität und Flexibilität, die für Forschung und Entwicklung notwendig ist.

Gonzalez/Denisi (2009) untersuchen an 26 Filialen einer US-amerikanischen Restaurantkette, inwieweit der Zusammenhang zwischen Gender Diversity auf Team- beziehungsweise Organisationsebene und der Firmenleistung (gemessen anhand verschiedener finanzieller Indikatoren) durch das „Diversity Climate“ moderiert wird ${ }^{2}$. Letzteres wurde definiert als aggregierte Wahrnehmung der Belegschaft hinsichtlich formaler Strukturen sowie informeller Werte innerhalb der Organisation im Hinblick auf Diversity. Dabei geht es insbesondere um die Wahrnehmung einer fairen Behandlung aller sozialen Gruppen durch die Organisation und den Ein- oder Ausschluss bestimmter Personengruppen mit diversem Hintergrund.

\footnotetext{
${ }^{1} \mathrm{Vgl}$. Richard/Ford/Ismail (2006).

${ }^{2}$ Vgl. Gonzalez/Denisi (2009).
} 
Die Ergebnisse der Untersuchung zeigen, dass der Zusammenhang zwischen Gender Diversity und Team- bzw. Organisationserfolg invers-U-förmig ist, wenn das Diversity Climate als günstig (,,supportive") wahrgenommen wurde. Unter dieser Bedingung hat demnach vor allem ein mittleres Niveau an Gender Diversity einen positiven Effekt auf Team- bzw. Organisationserfolg. Während es einleuchtend scheint, dass ein niedriges Niveau an Gender Diversity nicht ausreicht, um im Sinne der Information/ Decision-Making-Perspektive positive Effekte freizusetzen, bleiben die Autoren die Erklärung dafür schuldig, dass dieser positive Effekt bei einem hohen Maß an Gender Diversity wieder sinkt. Wurde das Diversity Climate dagegen als ungünstig (,,adverse“) bewertet, manifestierte sich dies in einem U-förmigen Zusammenhang zwischen Gender Diversity und Firmenleistung: Unter dieser Bedingung wirken sich eine sehr geringe und eine sehr hohe Gender Diversity positiv auf den Team- bzw. Organisationserfolg aus. Eine sehr geringe Gender Diversity lässt Prozesse der sozialen Kategorisierung gar nicht erst aufkommen; bei einer sehr hohen Gender Diversity werden negative Effekte eines ungünstigen Diversity Climate abgepuffert. Insbesondere die letztere Argumentation erscheint schwer nachvollziehbar, zumal die Autoren nicht erläutern, was jeweils unter geringer, mittlerer und hoher Gender Diversity zu verstehen ist.

In Anknüpfung an die Unternehmenskultur als potenziell moderierenden Effekt untersuchen Richard et al. (2004) in 153 US-amerikanischen Banken die unternehmerische Orientierung als Moderator $^{3}$. Die Autoren bestätigen zunächst einen U-förmigen Zusammenhang zwischen Cultural Diversity (bezogen auf Ethnizität und Geschlecht) und Organisationserfolg. Wie oben erläutert, ist ein negativer Effekt von Gender Diversity vor allem bei einem mittleren Diversity-Grad zu erwarten. Die Art dieses Zusammenhangs wird jedoch durch unternehmerische Risikobereitschaft moderiert. Bei hoch ausgeprägter unternehmerischer Risikobereitschaft ergibt sich ein invers-U-förmiger Zusammenhang: Bei geringer Gender Diversity sind die Perspektiven der Teammitglieder nicht heterogen genug, um adäquate Entscheidungen zu treffen. Bei hoch ausgeprägter Gender Diversity lassen Verhaltensweisen nach, die auf Konsens und soziale Kohäsion ausgerichtet sind und Entscheidungen werden zu langsam getroffen, so dass die Risikobereitschaft des Unternehmens ebenfalls nicht genügend unterstützt wird. Bei mittlerer Gender Diversity wird dagegen ein Niveau an

\footnotetext{
${ }^{3} \mathrm{Vgl}$. Richard et al. (2004).
} 
Aufgabenkonflikten erreicht, das der Risikobereitschaft des Unternehmens in idealer Weise entgegenkommt.

\section{Teameigenschaften}

Hirschfeld et al. (2005) können in ihrer Studie von 92 Teams im US-amerikanischen MilitärKontext einen Moderationseffekt der Teamaufgabe bestätigen ${ }^{4}$. So führt ein höherer Frauenanteil im Sinne der Information/Decision-Making-Perspektive zwar zu besseren Problemlösungsfähigkeiten der Arbeitsgruppen; bei physisch anstrengenden Aufgaben zeigt sich jedoch, dass ein höherer Frauenanteil zu etwas schlechteren Resultaten beiträgt. Die Autoren begründen ihre Hypothese mit der Benachteiligung von Frauen bei solchen Aufgaben, die körperliche Kraft und Ausdauer verlangen.

Wegge et al. (2008) untersuchen an 4.538 Steuerfachangestellten in 222 Arbeitseinheiten deutscher Steuerbehörden einen möglichen Effekt der Teamgröße auf den Zusammenhang zwischen Gender Diversity und Teamleistung - gemessen als Zeit zur Bearbeitung von Steuererklärungen in Finanzämtern ${ }^{5}$. Sie erwarten, dass das Merkmal Gender in großen Teams eine höhere Salienz besitzt als in kleinen Teams und dass Gender-spezifisches Verhalten daher ausgeprägter ist. Daher sollte der Zusammenhang zwischen Gender Diversity und Performanz in großen Teams positiver ausfallen als in kleinen Teams. Entgegen dieser Erwartung zeigt sich jedoch, dass Gender Diversity im Fall großer Teams einen signifikant negativen Zusammenhang mit der Bearbeitungsgeschwindigkeit aufweist während im Fall kleiner Teams kein Zusammenhang besteht.

\section{Diversity-Management}

Die Studie von Shaw (2004) an 390 australischen Studierenden in 64 Projektteams kommt zu dem Ergebnis, dass die (per Selbsteinschätzung gemessenen) „Diversity Management“-Fähigkeiten von Studierenden deren Studienleistungen in geschlechterdiversen Lerngruppen nicht per se positiv beeinflussen ${ }^{6}$. Vielmehr kommt der Autor zu dem Schluss, dass gute Diversity Management-

\footnotetext{
${ }^{4} \mathrm{Vgl}$. Hirschfeld et al. (2005).

${ }^{5}$ Vgl. Wegge et al.; Joshi/Roh (2009).

${ }^{6}$ Vgl. Shaw (2004).
} 
Fähigkeiten die Studienleistungen nur dann positiv beeinflussen, wenn das Geschlecht des jeweiligen Studierenden entweder dem der Mehrheit des Teams entspricht oder das Geschlechterverhältnis im Team ausgewogen ist. Ist ein Studierender hingegen Teil der geschlechtlichen Minderheit in einem Team, so wirken sich gute Diversity Management-Fähigkeiten - je nach Leistungskriterium - gar nicht oder negativ auf die Studienleistungen aus. Als mögliche Erklärung gibt Shaw (2004) an, dass die gemessenen Diversity-Management-Fähigkeiten eher genereller Natur sind und für Gender Diversity spezifischere Fähigkeiten erforderlich wären.

Widersprüchlich sind die von Choi/Rainey (2010) berichteten Befunde ${ }^{7}$. Wird als Operationalisierung für Diversity Management das in der Belegschaft wahrgenommene Ausmaß von Diversity Management verwendet, kann der erwartete Moderationseffekt der Beziehung zwischen Gender Diversity und Performanz nicht bestätigt werden; wird dagegen die Beschwerderate im Zusammenhang mit Gleichstellungsmaßnahmen (EEO-complaints- equal employment opportunity program-) verwendet, so zeigt sich ein unerwarteter Effekt: In Unternehmen mit hoher EEO-Beschwerderate ist der Zusammenhang zwischen Gender Diversity und Performanz positiv. Als Erklärung vermuten die Autoren lediglich, dass Gender Diversity in geringerem Umfang als angenommen zu Konflikten und Koordinationskosten führt und daher ein effektives Diversity-Management weniger notwendig ist.

\footnotetext{
${ }^{7}$ Vgl. Choi/Rainey (2010).
} 
Tabelle 1: Zusammenfassung der in der Analyse berücksichtigten Studien

\begin{tabular}{|c|c|c|c|c|c|}
\hline Autor(en) & Typ & Abhängige Variable & Stichprobe/Kontext & Ergebnis(se) & Besonderheiten und Limitationen \\
\hline Ali et al. (2009) & Primärstudie & $\begin{array}{l}\text { Organizational Per- } \\
\text { formance (Mitarbeiter- } \\
\text { Produktivität, Eigen- } \\
\text { kapitalrendite) }\end{array}$ & $\begin{array}{l}213(2002) \text { bzw. } 209 \\
\text { (2005) an der Australi- } \\
\text { schen Wertpapierbörse } \\
\text { gelistete Unternehmen; } \\
\text { Daten zur Performanz von } \\
2007\end{array}$ & $\begin{array}{l}\text { Sektor als Moderator bestä- } \\
\text { tigt (bei gering bis mittelstark } \\
\text { ausgeprägter Gender Diversi- } \\
\text { ty): } \\
\text { - Im Dienstleistungsbereich } \\
\text { positive Effekte von Gender } \\
\text { Diversity stärker } \\
\bullet \ddot{Y} \text { Im Produktionsbereich } \\
\text { negative Effekte von Gender } \\
\text { Diversity stärker }\end{array}$ & $\begin{array}{l}\text { - Große Aussagekraft der Ergebnisse } \\
\text { durch hohe Varianz der Gender Diver- } \\
\text { sity: Frauenanteil mind. zwischen 5\% } \\
\text { und 99\% } \\
\text { - Längsschnittdaten: } 2 \text { bzw. } 5 \text { Jahre } \\
\text { Zeitunterschied in der Messung von } \\
\text { UV und AV }\end{array}$ \\
\hline $\begin{array}{l}\text { Carter et al. } \\
(2010)\end{array}$ & Primärstudie & $\begin{array}{l}\text { Firm's Financial Per- } \\
\text { formance }\end{array}$ & $\begin{array}{l}\text { 641 Firmen, die zwischen } \\
1998 \text { und } 2002 \text { mindestens } \\
\text { einmal im Standard \& } \\
\text { Poor's 500-Index (Aktien- } \\
\text { index, der die } 500 \text { größten } \\
\text { börsennotierten US- } \\
\text { Unternehmen umfasst) } \\
\text { aufgelistet waren }\end{array}$ & $\begin{array}{l}\text { Positive Effekte von Gender } \\
\text { Diversity auf die Unterneh- } \\
\text { mensperformanz nicht bestä- } \\
\text { tigt }\end{array}$ & $\begin{array}{l}\text { - Besonderheit: Kein Zusammen- } \\
\text { hang zwischen Gender Diversity im } \\
\text { Vorstand und Unternehmensperfor- } \\
\text { manz angenommen }\end{array}$ \\
\hline Choi (2009) & Primärstudie & $\begin{array}{l}\text { Job Satisfaction } \\
\text { Turnover Intention } \\
\text { (jeweils auf Individu- } \\
\text { alebene) }\end{array}$ & $\begin{array}{l}\text { 67 US-Behörden (ent- } \\
\text { spricht ca. } 150.000 \text { Ange- } \\
\text { stellten im öffentlichen } \\
\text { Dienst); Datenquellen: } \\
\text { FHCS (Federal Human } \\
\text { Capital Survey) und CPDF } \\
\text { (Central Personnel Data } \\
\text { File) }\end{array}$ & $\begin{array}{l}\text { Negative Effekte von Gender } \\
\text { Diversity auf das Organizati- } \\
\text { onal Citizenship Behavior der } \\
\text { Gruppenmitglieder bestätigt }\end{array}$ & $\begin{array}{l}\text { - Die Turnover Intention kann sich } \\
\text { erheblich vom tatsächlichen Turnover } \\
\text { unterscheiden und ist somit nicht au- } \\
\text { tomatisch mit Verlusten für eine Or- } \\
\text { ganisation gleichzusetzen. }\end{array}$ \\
\hline
\end{tabular}




\begin{tabular}{|c|c|c|c|c|c|}
\hline $\begin{array}{l}\text { Choi/Rainey } \\
(2010)\end{array}$ & Primärstudie & $\begin{array}{l}\text { Organizational Per- } \\
\text { formance (Wahrneh- } \\
\text { mung der Angestell- } \\
\text { ten) }\end{array}$ & \begin{tabular}{|l|}
67 US-Behörden (ent- \\
spricht ca. 150.000 Ange- \\
stellten im öffentlichen \\
Dienst); Datenquellen: \\
FHCS (Federal Human \\
Capital Survey) und CPDF \\
(Central Personnel Data \\
File)
\end{tabular} & $\begin{array}{l}\text { - Dauer der Zusammenar- } \\
\text { beit als verstärkender Mode- } \\
\text { rator bestätigt } \\
\text { • Mitarbeiterorientierte Un- } \\
\text { ternehmenskultur als Mode- } \\
\text { rator nicht bestätigt }\end{array}$ & $\begin{array}{l}\text { - Subjektive Evaluation des Diver- } \\
\text { sity Managements und der Organisati- } \\
\text { onsleistung könnte zu methodenindu- } \\
\text { ziertem Bias führen }\end{array}$ \\
\hline $\begin{array}{l}\text { Gonzalez/Denisi } \\
(2009)\end{array}$ & Primärstudie & $\begin{array}{l}\text { Firm's Financial Per- } \\
\text { formance }\end{array}$ & $\begin{array}{l}\text { 26 Filialen einer regionalen } \\
\text { Restaurant-Kette, } 271 \mathrm{Be}- \\
\text { fragte }\end{array}$ & $\begin{array}{l}\text { Diversity Climate (DC) als } \\
\text { Moderator eines kurvilinea- } \\
\text { ren Zusammenhangs: } \\
\text { • Bei günstigem DC invers- } \\
\text { U-förmig bestätigt } \\
\text { - Bei ungünstigem DC U- } \\
\text { förmig bestätigt }\end{array}$ & $\begin{array}{l}\text { - Relativ hohe Gender Diversity im } \\
\text { Sample (gesamt: 56,3\% Frauen) } \\
\text { - Gewerblicher, nicht hochqualifi- } \\
\text { zierter Kontext (in der Diversity- } \\
\text { Forschung eher selten) } \\
\text { - Erhöhte Reliabilität in der Mes- } \\
\text { sung der Leistung von Unternehmens- } \\
\text { einheiten durch drei numerische Ma- } \\
\text { Be: Produktivität der Angestellten, } \\
\text { Rendite und Renditebeteiligung } \\
\text { - Geringe Rücklaufquote der Be- } \\
\text { fragten (27,2\%) } \rightarrow \text { mögliche Verzer- } \\
\text { rung durch häufigere Antworten von } \\
\text { mit dem DC unzufriedenen Mitarbei- } \\
\text { tern }\end{array}$ \\
\hline Herring (2009) & Primärstudie & $\begin{array}{l}\text { Organizational Per- } \\
\text { formance }\end{array}$ & $\begin{array}{l}506 \text { Unternehmen aus dem } \\
1996 \text { und } 1997 \text { National } \\
\text { Organizations Survey }\end{array}$ & $\begin{array}{l}\text { Positiver Zusammenhang } \\
\text { zwischen Gender Diversity } \\
\text { und Performanz bestätigt }\end{array}$ & $\begin{array}{l}\text { - Eingeschränkte Varianz der Da- } \\
\text { ten: homogen männliche und ge- } \\
\text { schlechterparitätische, jedoch keine } \\
\text { homogen weiblichen Teams in der } \\
\text { Stichprobe vorhanden } \\
\text { - Ungewöhnliches Maß für Gender } \\
\begin{array}{l}\text { Diversity: AID (Asymmetrical Index } \\
\text { of Diversity) }\end{array}\end{array}$ \\
\hline
\end{tabular}




\begin{tabular}{|c|c|c|c|c|c|}
\hline $\begin{array}{l}\text { Hirschfeld et al. } \\
\text { (2005) }\end{array}$ & Primärstudie & Team Task Proficiency & $\begin{array}{l}92 \text { neu gebildete Teams im } \\
\text { Militärbereich }\end{array}$ & $\begin{array}{l}\text { Moderationseffekt der Team- } \\
\text { aufgabe: } \\
\text { - Höherer Frauenanteil führt } \\
\text { zu besseren Problemlösungs- } \\
\text { fähigkeiten bestätigt } \\
\text { - Höherer Frauenanteil führt } \\
\text { zu schlechteren Resultaten } \\
\text { bei physisch anstrengenden } \\
\text { Aufgaben bestätigt }\end{array}$ & $\begin{array}{l}-\quad \text { Eingeschränkte Varianz, da kein } \\
\text { rein männlich besetztes Team und } \\
\text { geringer Frauenanteil in den Teams } \\
\text { (zwischen } 8 \text { und 31\%) }\end{array}$ \\
\hline $\begin{array}{l}\text { Jackson et al. } \\
(2003)\end{array}$ & Review & Performance & $\begin{array}{l}63 \text { Studien (1997-2002), } \\
\text { davon } 23 \text { zu Gender Diver- } \\
\text { sity und Performance; } \\
\text { Kontexte: Produktion, Top } \\
\text { Management, Vorstände, } \\
\text { zeitlich begrenzte Projekt- } \\
\text { teams, Fachressorts, Abtei- } \\
\text { lungen, ganze Organisatio- } \\
\text { nen, Studierendenteams }\end{array}$ & $\begin{array}{l}\text { Teilweise positive, teilweise } \\
\text { negative und teilweise insig- } \\
\text { nifikante Ergebnisse }\end{array}$ & $\begin{array}{l}\text { - Nur Studien einbezogen, in denen } \\
\text { „compositional effects“ (Einfluss kol- } \\
\text { lektiver Diversity-Eigenschaften auf } \\
\text { Individuen) untersucht werden } \\
\text { - } \quad \text { Einbezogene Studien untersuchen } \\
\text { häufig nur demographische Diversity } \\
\text { (und nicht direkt oder ergänzend die } \\
\text { daraus resultierend angenommene } \\
\text { kognitive Diversity) }\end{array}$ \\
\hline $\begin{array}{l}\text { Joshi/Roh } \\
\text { (2009) }\end{array}$ & Meta-Analyse & $\begin{array}{l}\text { Performance (Zieler- } \\
\text { reichung von Teams) }\end{array}$ & $\begin{array}{l}39 \text { Studien (8757 Teams), } \\
\text { die zwischen } 1992 \text { und } \\
2009 \text { durchgeführt wurden }\end{array}$ & $\begin{array}{l}\text { - Kein signifikanter direkter } \\
\text { Effekt } \\
\text { • Beschäftigungssektor } \\
\text { (Dienstleistung, aber nicht } \\
\text { Produktion und Hochtechno- } \\
\text { logie) als verstärkender Mo- } \\
\text { derator bestätigt }\end{array}$ & $\begin{array}{l}\text { - Konsistente Messung von Gender } \\
\text { Diversity in den einbezogenen Studien } \\
\text { durch den Blau-Index oder den } \\
\text { Teachman's Entropy Index } \\
\text { - Teaminnovation wurde unter } \\
\text { Performanz im weiteren Sinne einge- } \\
\text { schlossen }\end{array}$ \\
\hline
\end{tabular}




\begin{tabular}{|c|c|c|c|c|c|}
\hline & & & & $\begin{array}{l}\text { - Ausgeglichenes Ge- } \\
\text { schlechterverhältnis in der } \\
\text { jeweiligen Branche als ver- } \\
\text { stärkender Moderator bestä- } \\
\text { tigt } \\
\text { - Teaminterdependenz als } \\
\text { verstärkender Moderator } \\
\text { nicht bestätigt } \\
\text { - Dauer der Zusammenar- } \\
\text { beit im Team als hemmender } \\
\text { Moderator bestätigt }\end{array}$ & $\begin{array}{l}\text { - Explizite Untersuchung der Kon- } \\
\text { textfaktoren von Diversity wegen } \\
\text { diesbezüglichem Forschungsbedarf } \\
\text { (jedoch teilweise keine Kodierung von } \\
\text { Kontextfaktoren möglich aufgrund } \\
\text { ungenügender Beschreibung in den } \\
\text { Primärstudien) } \\
\text { - Beschränkung auf Studien in US- } \\
\text { amerikanischen Organisationen } \rightarrow \\
\text { eingeschränkte Generalisierbarkeit der } \\
\text { Ergebnisse, da ggf. kulturelle oder } \\
\text { landesspezifische Kontextfaktoren } \\
\text { bedeutenden Einfluss besitzen können }\end{array}$ \\
\hline $\begin{array}{l}\text { Kochan et al. } \\
(2003)\end{array}$ & $\begin{array}{l}\text { Review und } \\
\text { empirische } \\
\text { Studie }\end{array}$ & Business Performance & $\begin{array}{l}\text { Feldstudie mit vier Unter- } \\
\text { nehmen; Erhebung qualita- } \\
\text { tiver und quantitativer Da- } \\
\text { ten sowie Rückgriff auf } \\
\text { Archivdaten; Branchen: } \\
\text { Informationsverarbeitung, } \\
\text { Finanzdienstleistungen, } \\
\text { Einzelhandel }\end{array}$ & $\begin{array}{l}\text { - Kein signifikanter direkter } \\
\text { Effekt } \\
\text { - Kundenorientierte Ge- } \\
\text { schäftsstrategie, mitarbeiter- } \\
\text { orientierte Organisationskul- } \\
\text { tur und ,diversity-focused } \\
\text { human resource practices“ als } \\
\text { verstärkende Moderatoren } \\
\text { bestätigt } \\
\text { - Teilnahme an speziellen } \\
\text { Diversity- } \\
\text { Schulungsprogrammen als } \\
\text { Moderator nicht bestätigt } \\
\text { - Moderierender Effekt des }\end{array}$ & $\begin{array}{l}\text { • Größtes Forschungsprojekt zum } \\
\text { Test des ,business case“ für Diversity } \\
\text { bis } 2003 \\
\text { • Branchenübergreifende Studie, } \\
\text { die keine branchenspezifischen Effek- } \\
\text { te von Diversity zeigt } \\
\text { - Ausschließlich Untersuchung von } \\
\text { Organisationen, die sich seit längerer } \\
\text { Zeit für Diversity einsetzen: mögliche } \\
\text { Verzerrung } \\
\text { - Reliable Messung von Perfor- } \\
\text { manz durch Einbezug vieler unter- } \\
\text { schiedlicher objektiver Performanz- } \\
\text { maße }\end{array}$ \\
\hline
\end{tabular}




\begin{tabular}{|c|c|c|c|c|c|}
\hline & & & & $\begin{array}{l}\text { Geschlechts der jeweiligen } \\
\text { Führungskraft: in Teams mit } \\
\text { männlichen Führungskräften } \\
\text { bestand kein Zusammenhang } \\
\text { zwischen Gender Diversity } \\
\text { und Performanz; in Teams } \\
\text { mit weiblichen Führungskräf- } \\
\text { ten negativer Zusammenhang } \\
\text { bestätigt }\end{array}$ & $\begin{array}{l}\text { - Keine einheitliche Messung von } \\
\text { Gender Diversity in den vier unter- } \\
\text { suchten Organisationen (durch Rück- } \\
\text { griff auf unterschiedliche Firmenar- } \\
\text { chivdaten) }\end{array}$ \\
\hline $\begin{array}{l}\text { Leonard et al. } \\
\text { (2004) }\end{array}$ & Primärstudie & Store Performance & $\begin{array}{l}70.000 \text { Beschäftigte in } 700 \\
\text { Einzelhandelsgeschäften }\end{array}$ & $\begin{array}{l}\text { Geschlechterdiverse Kund- } \\
\text { schaft als verstärkender Mo- } \\
\text { derator nicht bestätigt }\end{array}$ & $\begin{array}{l}\text { • } \quad \text { Höchste Fallzahl der analysierten } \\
\text { Primärstudien: } 700 \text { (Geschäfte) } \\
\text { - Stabilität der Randbedingungen, } \\
\text { v.a. durch einheitlichen Arbeitgeber } \\
\text { (Kette) mit konstanten HR-Praktiken } \\
\text { gewährleistet } \\
\text { @ Hohe Varianz der UV: gesamte } \\
\text { Bandbreite an Frauenanteilen in den } \\
\text { Filialen gegeben } \\
\text { - Mangelhafte Messung der poten- } \\
\text { ziellen Kundschaft durch Zensusdaten } \\
\text { (Wohnbevölkerung der Umgebung } \\
\text { entspricht nicht potenzieller Kund- } \\
\text { schaft) }\end{array}$ \\
\hline $\begin{array}{l}\text { Pitts/Wise } \\
(2010)\end{array}$ & Review & Performance & $\begin{array}{l}89 \text { Studien im öffentlichen } \\
\text { Sektor, von denen } 44 \text { Gen- } \\
\text { der Diversity untersuchen } \\
\text { (seit 2000) }\end{array}$ & $\begin{array}{l}\text { Positiver Zusammenhang } \\
\text { bestätigt }\end{array}$ & $\begin{array}{l}\text { - Verwendung sehr unterschiedli- } \\
\text { cher und teilweise wenig vergleichba- } \\
\text { rer Maße für Performanz, zum Teil nur } \\
\text { Proxies oder Antezedenten von Per- } \\
\text { formanz (Bsp.: Wahrnehmung der } \\
\text { Organisationsressourcen durch die } \\
\text { Angestellten) }\end{array}$ \\
\hline
\end{tabular}




\begin{tabular}{|c|c|c|c|c|c|}
\hline & & & & & $\begin{array}{l}\text { - Referierte Studien zeigen, dass } \\
\text { Diversity-Forschung im öffentlichen } \\
\text { Sektor weniger weit fortgeschritten ist } \\
\text { als die allgemeine Diversity- } \\
\text { Forschung, da im öffentlichen Bereich } \\
\text { noch vorrangig Haupteffekte (statt } \\
\text { Moderatoren/Mediatoren) untersucht } \\
\text { werden } \\
\text { - Inwieweit eine Differenzierung } \\
\text { zwischen öffentlichem und privatwirt- } \\
\text { schaftlichem Sektor in der Diversity- } \\
\text { Forschung sinnvoll ist bzw. sich For- } \\
\text { schungsergebnisse durch mögliche } \\
\text { Charakteristika des öffentlichen } \\
\text { Dienstes maßgeblich unterscheiden, } \\
\text { wird von den Autoren nicht weiter } \\
\text { diskutiert } \\
\text { • ,Diversity' der Untersuchungsde- } \\
\text { signs: Diversity ist nur teilweise UV, } \\
\text { ein empirisches, hypothesentestendes } \\
\text { Design weisen nicht alle Studien auf }\end{array}$ \\
\hline $\begin{array}{l}\text { Richard et al. } \\
\text { (2004) }\end{array}$ & Primärstudie & Performance & $\begin{array}{l}153 \text { Banken (mit durch- } \\
\text { schnittlich } 154 \text { Angestell- } \\
\text { ten) }\end{array}$ & $\begin{array}{l}\text { Unternehmerische Orientie- } \\
\text { rung als Moderator: Bei hoch } \\
\text { ausgeprägter unternehmeri- } \\
\text { scher Risikobereitschaft in- } \\
\text { vers-U-förmiger Zusammen- } \\
\text { hang bestätigt }\end{array}$ & $\begin{array}{l}\text { • Um für branchenspezifische Ef- } \\
\text { fekte zu kontrollieren, wurde die Un- } \\
\text { tersuchung auf eine Branche be- } \\
\text { schränkt, daher eingeschränkte Gene- } \\
\text { ralsierbarkeit }\end{array}$ \\
\hline $\begin{array}{l}\text { Richard et al. } \\
\text { (2006) }\end{array}$ & Primärstudie & $\begin{array}{l}\text { Organizational Per- } \\
\text { formance (objektive } \\
\text { Maße) }\end{array}$ & 79 Banken & $\begin{array}{l}\text { Geringe Kontrollspanne und } \\
\text { frühe Phase im Lebenszyklus } \\
\text { als verstärkende Moderatoren } \\
\text { bestätigt }\end{array}$ & $\begin{array}{l}\text { - Um für branchenspezifische Ef- } \\
\text { fekte zu kontrollieren, wurde die Un- } \\
\text { tersuchung auf eine Branche be- } \\
\text { schränkt, daher eingeschränkte Gene- } \\
\text { ralisierbarkeit. }\end{array}$ \\
\hline
\end{tabular}




\begin{tabular}{|c|c|c|c|c|c|}
\hline Shaw (2004) & Primärstudie & Studierendenleistungen & $\begin{array}{l}\text { Längsschnittstudie mit } 390 \\
\text { Studierenden }\end{array}$ & $\begin{array}{l}\text { Diversity Management- } \\
\text { Fähigkeiten von Studierenden } \\
\text { als verstärkender Moderator } \\
\text { nicht bestätigt }\end{array}$ & $\begin{array}{l}\text { • } \quad \text { Besonderheit: Längsschnittstudie, } \\
\text { dadurch kausale Rückschlüsse mög- } \\
\text { lich } \\
\text { • } \quad \text { Pädagogische Perspektive der } \\
\text { Studie, keine Evaluation beruflicher } \\
\text { Leistungen } \\
\text { - Studie auf Individualebene } \\
\text { - } \quad \text { Einbezug der „,Gender-Position“ } \\
\text { der Studierenden in ihrer Gruppe: } \\
\text { „dominant member“, „balanced mem- } \\
\text { ber“ oder „,balanced member“ }\end{array}$ \\
\hline $\begin{array}{l}\text { Shore et al. } \\
(2009)\end{array}$ & Review & Performance & $\begin{array}{l}\text { Gender Diversity-Literatur } \\
\text { seit } 2000 \text { als Grundlage }\end{array}$ & Inkonsistente Befunde & $\begin{array}{l}\text { - Die verschiedenen Operationali- } \\
\text { sierungen von Gender Diversity und } \\
\text { Performanz werden nicht referiert - } \\
\text { allerdings die daraus folgende einge- } \\
\text { schränkte Vergleichbarkeit } \\
\text { - Appell, neue theoretische Ansätze } \\
\text { zu einer positiven oder neutralen Wir- } \\
\text { kung von Diversity zu nutzen }\end{array}$ \\
\hline $\begin{array}{l}\text { Wegge et al. } \\
(2008)\end{array}$ & Primärstudie & Performance & $\begin{array}{l}222 \text { Arbeitseinheiten in } \\
\text { deutschen Steuerbehörden } \\
\text { (4538 Steuerfachangestell- } \\
\text { te) }\end{array}$ & $\begin{array}{l}\text { Teamgröße als verstärkender } \\
\text { Moderator nicht bestätigt }\end{array}$ & $\begin{array}{l}-\quad \text { Untersuchung nur einer Berufs- } \\
\text { gruppe } \rightarrow \text { Wirkung von für diese Tä- } \\
\text { tigkeit spezifischen Kontextfaktoren } \\
\text { möglich } \\
\text { • Im Untersuchungskontext keine } \\
\text { männlich dominierten Teams, jedoch } \\
\text { weiblich dominierte und solche mit } \\
\text { ausgeglichenem Geschlechterverhält- } \\
\text { nis }\end{array}$ \\
\hline
\end{tabular}




\begin{tabular}{|l|l|l|l|} 
& & & $\begin{array}{l}\text { Gender Diversity wurde stellver- } \\
\text { tretend für die Salienz (wahrgenom- } \\
\text { mene Auffälligkeit) von Gender ver- } \\
\text { wendet }- \text { letztere also nicht direkt } \\
\text { gemessen }\end{array}$ \\
\hline
\end{tabular}

